Check for updates

Cite this: Mater. Adv., 2021

2, 1507

Received 23rd October 2020

Accepted 28th January 2021

DOI: 10.1039/d0ma00829j

rsc.li/materials-advances

\title{
Recent advancements in coinage metal nanostructures and bio-applications
}

\begin{abstract}
Si Yin Tee and Enyi Ye (D)*
Coinage metals are best known for their monetary, adorning, and metallurgical values from a historical perspective, while their metal nanostructures and applications may be considered as products of modern science since the potential of nanotechnology has only been realized in recent years. Coinage metal nanostructures possess noteworthy properties such as size- and shape-dependent catalytic, optical, electrical, and chemical properties. Each of these attributes has inspired significant investigation toward the design and synthesis of coinage metal nanostructures as well as their potential application. For these reasons, this review summarizes the recent development of coinage metals such as $\mathrm{Cu}, \mathrm{Ag}$, $\mathrm{Au}$, and their bimetallic nanostructures, particularly using solution-based approaches. It covers various synthetic methodologies including standard protocols as well as recent strategies that have been implemented to improve the functionality by effectively controlling the size, morphology, and composition of the coinage metal nanostructures. It also highlights the applications of these coinage metal nanostructures in bio-applications such as antibacterial activities, surface-enhanced Raman scattering (SERS)-based detection, and electrochemical sensing. Moving forward, this review provides a brief discussion on the challenges and future directions of these promising metal nanostructures, which will be essentially useful in designing new nanostructures for better functionalities in the future.
\end{abstract}

\section{Introduction}

Metal nanostructures have been gaining tremendous interests owing to their extremely small feature size for wide applications, which is more superior to their bulk counterparts. The distinct property of nanoscale materials that differentiates them from their bulk properties is the increase in the surface area to volume ratio. The benefit of having a large surface area to volume ratio is that the percentage of surface atoms becomes significant, which can lead to an increase in the reactivity of the material. ${ }^{1,2}$ Therefore, their unique size and shape-dependent properties are promising for widespread commercial applications in the field of electronics, information storage, biomedical devices, as well as environmental and energy technologies. ${ }^{3-8}$ Among various metallic nanostructures, group 11 coinage metals (i.e., $\mathrm{Cu}, \mathrm{Ag}$, and $\mathrm{Au}$ ) have drawn a lot of interest and are being extensively studied by researchers within the large transitionmetal family. The modern scientific evaluation of metallic nanoparticles did not begin until Michael Faraday pioneered the preparation of colloidal Au solution by reducing Au salt with phosphorus in water. ${ }^{9}$ Later, Enüstün and Turkevich prepared spherical nanoparticles of $\mathrm{Au}$ and $\mathrm{Ag}$ with tunable sizes by the

Institute of Materials Research and Engineering, Agency for Science, Technology \& Research 2 Fusionopolis Way, Singapore, 138634, Singapore

E-mail:yeey@imre.a-star.edu.sg reduction of $\mathrm{Au}$ or $\mathrm{Ag}$ salts using citrate in aqueous solutions in 1960s. ${ }^{10}$ The design and synthesis of these coinage metals are crucial for fundamental research and technology development; to date, there have been multiple synthetic methods for developing different types of transition metal nanostructures with well-controlled dimensions. This includes hydro/solvothermal methods, seed/template-mediated synthesis, polyol synthesis, and electrochemical synthesis; the ultimate goal of these fabrication methods is to introduce new nanostructures and to improve the existing ones for better functionalities. At present, manipulation of the size, shape, architecture, composition, and structure at the nanoscale are essentially key factors in enhancing the properties of metal nanostructures and thus their potential applications.

In addition to monometallic nanostructures, bimetallic nanostructures are a new class of nanomaterials that may have better technological usefulness with distinct properties from those of individual atoms and molecules or bulk matter. Synergetic effects between two distinct elements make them superior to the monometallic counterpart, bringing superior or new physical/chemical properties and improved performances. ${ }^{11-15}$ The properties and the applicability of these bimetallic nanostructures not only depend on their size and shape but also on the combination of the component metals (composition) and their fine structure. Generally, bimetallic nanostructures are classified into three categories: alloyed structure, core-shell, 
and heterostructured, according to the mixing patterns of two different metals. The alloyed structure consists of the homogeneous distribution of the two types of metal atoms, which may be either ordered or randomly mixed, whereas the coreshell structure is made up of a shell of one type of metal ions surrounding a core of another metal. During the formation process, the metal ions that are reduced first form the inner core, while the other metal grows as a shell on the surface of the core, finally resulting in a core-shell structure. By controlling their core size and shell thickness, their properties can be well modulated. Nonetheless, under specific conditions, individual nucleation and growth of two types of metal atoms can independently occur to form the heterostructure. The mixed interface that they share during the growth process may play a significant role in controlling their behaviors.

The early scientific study of bimetallic nanoparticles was pioneered by Morriss and Collins, who synthesized the Au@Ag core-shell nanoparticles and studied their optical properties in the 1960s. ${ }^{16}$ The unique optical properties in these coinage nanostructures arise from the collective oscillation of conductive electrons (i.e., surface plasmon) as a result of the interaction of light waves and metallic surfaces. This fascinating finding has inspired further work on the development of bimetallic $\mathrm{Au}-\mathrm{Ag}$ nanostructures, particularly on their preparation, characterization, properties, and application. In this review article, we summarize the recent progress in solution-based synthetic routes for the design and preparation of metallic and bimetallic nanostructures of $\mathrm{Cu}, \mathrm{Ag}$, and $\mathrm{Au}$. In this aspect, more attention is focused on the tailoring of the particle size, shape, architecture, and composition of the nanostructured materials, which make them excellent candidates in antibacterial activities, SERS-based detection, and electrochemical sensing (Fig. 1). Lastly, we discuss the major

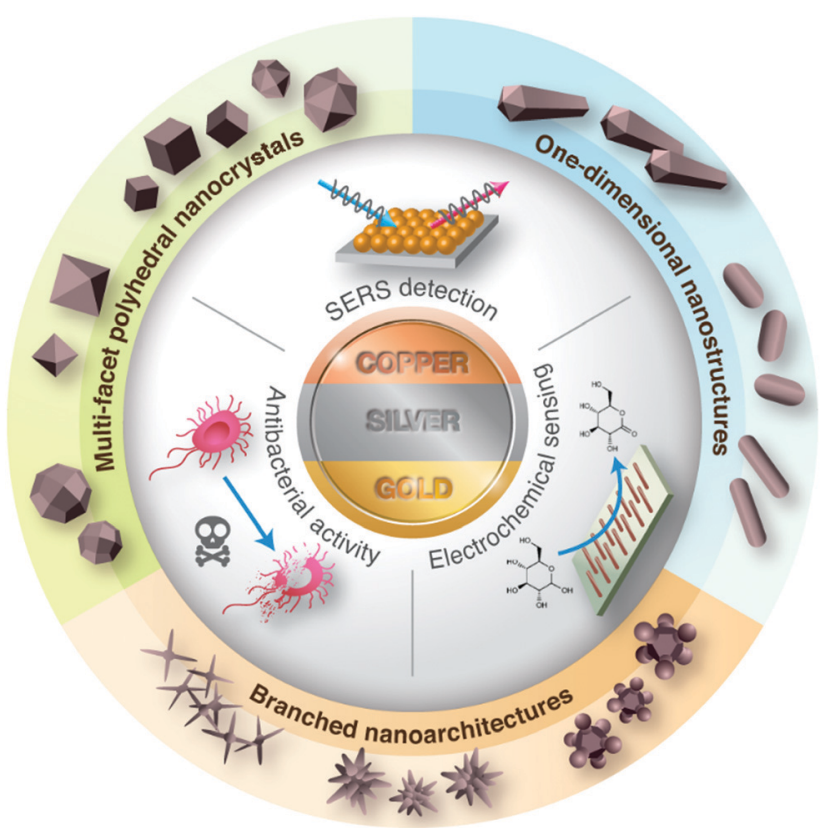

Fig. 1 General presentation of coinage metal nanostructures and their applications. challenges and future directions for these promising metal nanostructures.

\section{General synthetic methodology}

The research on nanostructured materials has greatly intensified due to the advancements of the synthetic procedure, which enable good control over the size and shape. There are two general approaches for metal nanostructure synthesis-topdown approaches and bottom-up assembly. Most top-down approaches are physical methods (i.e., milling and lithography), which involve the use of expensive synthetic pathways that are not suitable for large-scale industrial production. Also, this approach limits the control over the size, size distribution, and particle shape. On the other hand, bottom-up approaches such as wet chemical synthesis are far more commonly used in the synthesis of nanostructured materials, which enable the preparation of metal nanostructures in high yield and structural purity with varying size, shape, structure, composition, and surface chemistry. Typically, colloidal nanostructures are synthesized in the liquid phase by the reduction of metal salts with chemical reducing agents. ${ }^{17}$ The size and shape of the metal nanoparticles are controlled by the concentration, reducing agents, and capping agents. ${ }^{6,18}$ Spherical metal nanoparticles are usually prepared by the direct reduction of a metal salt with a strong reducing agent in the presence of capping agents. ${ }^{18}$ On the contrary, anisometric metal nanostructures are usually prepared using a seed-mediated growth approach, in which the preformed metal seed particles are added to a solution containing the metal salt, a weak-reducing agent, and a shape-directing agent. This concept prevents the formation of new agglomerates and provides the growth in an ordered and desired manner. ${ }^{18,19}$

The general path for the nucleation and growth of nanostructures from solution is summarized into five different stages, namely, (i) nucleation, (ii) growth, (iii) Ostwald ripening, (iv) surfactant capping, and (v) precipitation. The nucleation process represents the initial stage of any crystallization process, which refers to the production of monomers from the reaction of precursors. As the precursor decompose, the concentration of metal atoms increases gradually until it reaches a point of supersaturation, where the atoms start to aggregate into numerous small clusters and tend to quickly aggregate together to form larger, more thermodynamically stable particles. ${ }^{17,20}$ In most cases, Ostwald ripening happens at a later stage of crystal growth, which involves the dissolution of smaller particles and the consequent further growth of larger particles. During the formation process, a stabilizer or surfactant is added in order to prevent particle aggregation and mediate the growth process. Finally, the nanostructured products can be obtained by precipitation and further size-selective separation process can be performed to narrow the size distribution. With this colloidal synthesis method, nanostructures with various sizes, shapes, compositions, crystal structures, and surfactant coatings have been reported. For example, simple shapes such as spheres and cubes, elongated morphologies such as rods and wires, and 
more complicated faceted geometries such as plates and polyhedra have been fabricated. ${ }^{21-23}$ Hybrid nanostructures (i.e., heterostructures) as well as nanoscale alloys of metals with varying compositions have also been constructed. ${ }^{24,25}$

\subsection{Copper nanostructures}

$\mathrm{Cu}$ was one of the earliest metals to be exploited and is still considered as one of the important metals because of its excellent properties such as ductility, malleability, and conductivity. In comparison with another two coinage metals such as $\mathrm{Au}$ and $\mathrm{Ag}$, the preparation of stable and monodisperse $\mathrm{Cu}$ nanostructures with controllable sizes, shapes, and surface properties has been relatively limited due to the difficulty of reducing $\mathrm{Cu}$ salts into metallic $\mathrm{Cu}$ and their inherent propensity for surface oxidation in ambient conditions. ${ }^{26,27}$ To address the oxidation limitation, various polymers or surfactants have been used as reducing agents and capping agents, which serve as a protective layer for the preparation of $\mathrm{Cu}$ nanostructures in aqueous solutions.

2.1.1. Chemical reduction in aqueous media. An early study has shown that $\mathrm{Cu}$ nanoparticles could be prepared in an aqueous solution of anionic surfactant (i.e., sodium dodecyl sulphate) in a glove box to protect from oxidation. ${ }^{28}$ Also, the preparation of metallic $\mathrm{Cu}$ nanoparticles could be conducted in the ambient atmosphere via a polyol method using poly(vinylpyrrolidone), abbreviated as PVP. However, it was reported that $\mathrm{Cu}$ nanoparticles were surrounded by amorphous $\mathrm{CuO}$ on the $\mathrm{Cu}$ surface, showing the sign of oxidation. ${ }^{29}$ Besides, $\mathrm{Cu}$ nanowires at $60{ }^{\circ} \mathrm{C}$ in aqueous solution were synthesized at a large scale, starting from the formation of $\mathrm{Cu}(\mathrm{OH})_{2}$ precipitates upon mixing aqueous $\mathrm{Cu}$ salts and $\mathrm{NaOH}$ solutions, with the subsequent addition of ethylenediamine to control the product morphology and hydrazine as the reductant. ${ }^{30}$ Later, Rathmell et al. presented an aqueous phase approach for growing $\mathrm{Cu}$ nanowires from spherical $\mathrm{Cu}$ seeds with hydrazine as the reducing agent and ethylenediamine as the shape-directing agent to promote anisotropic growth. ${ }^{31}$ However, the drawback of this method is the poor dispersibility of the $\mathrm{Cu}$ nanowires in the aqueous solution caused by aggregation. Despite hydrazine being regarded as one of the reducing agents of choice in many reports, the cost and safety issues relating to this highly reactive chemical limit the feasible application.

Alternatively, glucose and ascorbic acid have been used as reducing agents to synthesize $\mathrm{Cu}$ nanostructures under elevated temperature for the reduction process to take place. ${ }^{32} \mathrm{Jin}$ et al. carried out the synthesis of $\mathrm{Cu}$ nanostructures using aqueousmedia reduction of $\mathrm{Cu}$ salts with glucose at $100{ }^{\circ} \mathrm{C}$. In this approach, hexadecylamine (HDA) was employed as the capping agent to produce $\mathrm{Cu}$ nanostructures with controlled shapes and good uniformity. ${ }^{26}$ The study suggested that HDA was an effective capping agent for $\mathrm{Cu}$ and the concentration of HDA was one of the key factors in controlling the morphology of the final product. By changing the concentration of HDA, the distribution of the single-crystal versus multiply-twinned seeds could be manipulated at the nucleation stage. In addition to the concentration of HDA, the reduction kinetics induced by glucose also have an important impact on the morphology of the final product. Both factors contributed to the formation of four different kinds of $\mathrm{Cu}$ nanostructures encased by (100) facets (i.e., pentagonal nanowires, tadpole-like nanowires, pentagonal bipyramids, and nanocubes) via the selective adsorption of HDA on the (100) facets. In a recent study, this HDA-assisted glucose reduction was further modified by $\mathrm{Fu}$ et al. to synthesize $\mathrm{Cu}$ nanowires in high yield via in situ seed-mediated two-step approach. ${ }^{33}$ The process involved the nucleation of the nanoseeds, in which $\mathrm{Cu}$ ions were reduced to form nondecahedral $\mathrm{Cu}$ nanodots and further used to promote the formation of decahedral multiply-twinned particles for the subsequent growth of $\mathrm{Cu}$ nanowires. The seed-mediated two-step approach restricted the spontaneous ripening of undesired $\mathrm{Cu}$ nanoparticle by-products by segregating the nucleation step at a relatively lower temperature, thereby improving the $\mathrm{Cu}$ nanowire production yield.

2.1.2. Chemical reduction in organic media. Despite the advantage that chemical synthesis in aqueous phase can be operated under ambient conditions, it remains a challenge to produce $\mathrm{Cu}$ nanostructures with narrow size distribution and high stability upon exposure to air. In this context, important progress has been made toward developing $\mathrm{Cu}$ nanostructures using a variety of organic phase-based methods. The following studies describe the synthesis of $\mathrm{Cu}$ nanostructures assisted by a variety of capping agents to maneuver the surface energies and growth rates of different facets and, therefore, the shape taken by a nanocrystal. For example, this has been demonstrated by Mott et al. to synthesize nanorods and nanocubes by controlling the reaction temperature using amine and acid capping agents in organic solvents. ${ }^{34}$ The morphologycontrolled synthesis was associated with the initial formation of a seed precursor as well as the facet-specific capping agents on specific nanocrystal facets that control the growth direction of the crystal facets selectively. Another synthetic strategy has been explored by Cui $e t a l$. to develop five-fold $\mathrm{Cu}$ nanowires by utilizing tris(trimethylsilyl)silane as a mild reducing reagent. ${ }^{35}$ In this approach, the mild reducing power of tris(trimethylsilyl) silane contributed significantly to slowing down the reduction kinetics, which, as a result, permitted time for the nucleation of the five-twinned pentagonal nanoparticles as the seeds and the subsequent growth of $\mathrm{Cu}$ nanowires. The silane approach also used oleylamine in the synthesis as a coordinating ligand for the $\mathrm{Cu}^{\mathrm{II}}$ precursor as well as a capping ligand on the $\mathrm{Cu}$ nanowire surface. To improve the dispersion stability, oleic acid was employed in the reaction as a supplementary ligand to prevent the aggregation of $\mathrm{Cu}$ nanowires at elevated temperatures.

In contrast to these approaches, our research group prepared the penta-twinned $\mathrm{Cu}$ nanowires through the controlled disproportionation of $\mathrm{Cu}^{\mathrm{I}}$ facilitated by oleylamine and the subsequent disproportionation of the $\mathrm{Cu}^{\mathrm{I}}$ complexes into metallic $\mathrm{Cu}^{\circ}$ and bivalent $\mathrm{Cu}^{\mathrm{II}}$ (Fig. 2). ${ }^{36}$ In addition, these oleylamine-coated $\mathrm{Cu}$ nanowires could self-assemble by surface ligand exchange with trioctylphosphine. The trioctylphosphine-coated $\mathrm{Cu}$ nanowires also served to improve the oxidation resistance and allowed them to be well stored in air. The disproportionation reaction 

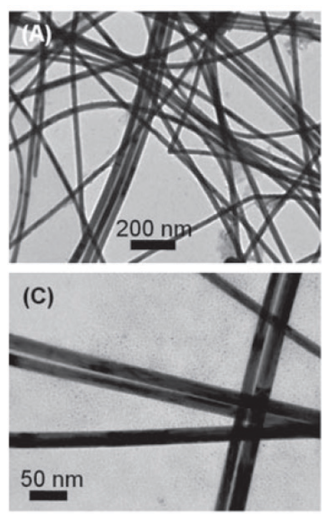

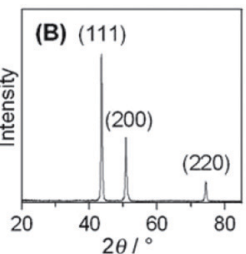

(D)

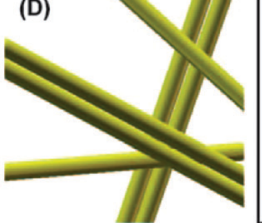

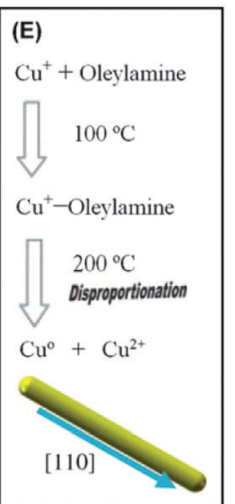

Fig. 2 Fabrication of oleylamine-coated Cu nanowires by the disproportionation reaction. (A) TEM image and (B) XRD pattern of $\mathrm{Cu}$ nanowires. (C) Magnified TEM image and (D) schematic formation of $\mathrm{Cu}$ nanowires. (E) Schematic formation of $\mathrm{Cu}$ nanowires via the disproportionation reaction. Reproduced from ref. 36 with permission from Wiley-VCH.

route was extended by Guo et al. to develop $\mathrm{Cu}$ nanospheres and $\mathrm{Cu}$ nanocubes using trioctylphosphine and trioctylphosphine oxide as the capping agents, respectively. ${ }^{37}$ The shape directing effect of the different capping agents was based on their coordination effects to $\mathrm{Cu}^{+}$(Fig. 3). For instance, the stronger coordination effect of trioctylphosphine to $\mathrm{Cu}^{+}$inhibited the metallic ions from being rapidly reduced to $\mathrm{Cu}^{0}$, resulting in nucleation at a higher temperature. This eventually led to the formation of isotropous crystals, which made the seeds grow equally in all directions into spherical-shaped crystals. On the other hand, the weaker coordination effect of trioctylphosphine oxide to $\mathrm{Cu}^{+}$promoted the nucleation process to occur at a relatively lower temperature, leading to the (100) facets of the $\mathrm{Cu}$ crystal seeds selectively capped by the ligands. Consequently, the other facets grew at a faster rate than the (100) facets. The lower

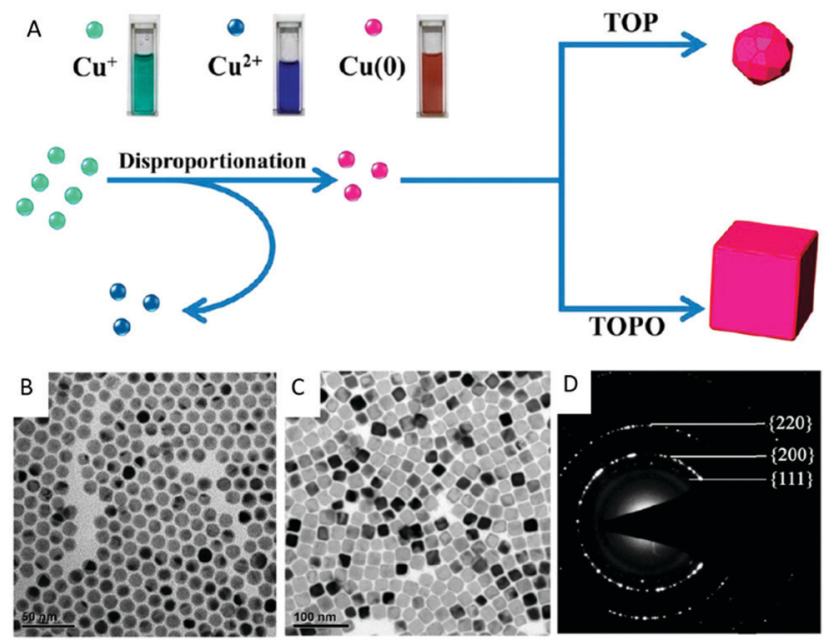

Fig. 3 (A) Schematic formation of Cu nanospheres and nanocubes via the disproportionation reaction route. TEM image of $(B)$ the $\mathrm{Cu}$ nanospheres with an average diameter of $12.6 \mathrm{~nm}$, (C) $\mathrm{Cu}$ nanocubes with an average edge length of $24.1 \mathrm{~nm}$, and (D) the corresponding SAED pattern. Reproduced from ref. 37 with permission from the American Chemical Society. reaction temperature allowed a slow growth rate and hence the different reaction kinetics contributed to the formation of different $\mathrm{Cu}$ nanostructures. Huang et al. developed two distinctly-shaped $\mathrm{Cu}$ nanostructures (i.e., nanocubes and nanowires) by employing different shape-controlling additives. ${ }^{38}$ The use of $\mathrm{NH}_{4} \mathrm{Cl}$ and $\mathrm{RuCl}_{3}$ additives in the synthetic process has been recognized as an effective approach to control the growth of $\mathrm{Cu}$ nanostructures. Specifically, $\mathrm{NH}_{4} \mathrm{Cl}$ was employed in the oxidative etching process to selectively etch away the seeds containing twin defects and provide single-crystal seeds to form the nanocubes. Conversely, the role of $\mathrm{RuCl}_{3}$ was to consume $\mathrm{O}_{2}$ in the reaction and thus prevent the twinned nuclei from being oxidized and etched. As a result, this protection enabled the continuous growth of the multiply twinned seeds and thereby induced the formation of $\mathrm{Cu}$ nanowires.

\subsection{Silver nanostructures}

Historically, Ag metal has been widely used in jewellery and ornaments, high-value tableware and utensils, trade, and as the basis of many monetary systems. In the biomedical field, Ag-containing materials have been used in wound dressings and in topical treatments to heal burn wounds. ${ }^{39,40}$ However, the emergence of several antibiotics has resulted in the declined usage of $\mathrm{Ag}$ compounds. The recent advances in nanotechnology have opened up a whole new strategy to use $\mathrm{Ag}$ metal against a wide array of pathogens due to the capability of modulating metals into their nanosized counterparts, which significantly alter the chemical, physical, and optical properties of Ag. In this respect, numerous physical and chemical approaches have been used for synthesizing Ag nanostructures, including evaporation-condensation, laser ablation, and chemical reduction. ${ }^{17,41-44}$ In physical processes, metal nanoparticles generated are found to be inefficient in the materials and energy used. ${ }^{45}$

2.2.1. Chemical reduction in aqueous media. Chemical reduction is the most commonly used approach for the synthesis of tunable $\mathrm{Ag}$ nanostructures in stable colloidal dispersions. Typically, reducing agents (i.e., sodium borohydride, sodium citrate, ascorbic acid, and polyols) are commonly used to reduce $\mathrm{Ag}^{+}$ions to $\mathrm{Ag}^{0}$ atoms, subsequently causing agglomeration into oligomeric clusters, and ultimately lead to the formation of colloidal Ag particles. ${ }^{46,47}$ It is well-known that protective agents/capping agents (i.e., thiols, amines, acids, and alcohols) are used to stabilize nanoparticles by absorbing or binding onto the nanoparticle surface, thus preventing their agglomeration and losing the surface properties. ${ }^{20,48,49}$ In addition, polymeric protective agents such as PVP, poly(vinyl alcohol), poly(ethylene glycol), poly(methacrylicacid), and polymethylmethacrylate have been employed to achieve good stabilization of the metallic nanostructures. ${ }^{46}$ With different synthetic approaches, various $\mathrm{Ag}$ nanostructures (i.e., nanorods, nanowires, nanotubes, nanoplates, nanospheres, and nanopolyhedrons) have been well controlled and synthesized successfully, as reported in the literature. ${ }^{50-58}$

The conventional borohydride-mediated or citrate-mediated reduction protocol (i.e., Lee-Meisel) have been developed for 
decades to reduce $\mathrm{Ag}$ ions to $\mathrm{Ag}$ nanoparticles but it can be a major challenge to obtain products with high monodispersity and stability. To address these limitations, numerous attempts have been made by many researchers to investigate the influence of reducing agents, capping agents, halides ions, and digestive ripening ligands in the synthesis of monodisperse $\mathrm{Ag}$ nanostructures of controlled size and shape. For instance, Agnihotri et al. adopted the co-reduction approach to produce Ag nanoparticles of different size ranges (5-100 nm), employing sodium borohydride as a primary reductant and trisodium citrate as the secondary reductant as well as stabilizing agent. ${ }^{59}$ The co-reduction approach provided a better balance between the nucleation and growth of $\mathrm{Ag}$ nanoparticles; therefore, the synthesis of size controlled Ag nanoparticles could be achieved. Li et al. developed a method to produce monodisperse, quasispherical Ag nanoparticles in the aqueous solution by reducing $\mathrm{AgNO}_{3}$ with both citrate and ascorbic acid in the presence of iodide ions. $^{60}$ To obtain uniform quasi-spherical Ag nanoparticles, ascorbic acid was used to promote fast nucleation in order to inhibit secondary nucleation due to the slow reduction of $\mathrm{Ag}$ ions by citrate. On the other hand, iodide ions were employed as strong absorbates to tailor the growth of $\mathrm{Ag}$ into a quasi-spherical shape via its preferential adsorption on the (111) facets. Yang et al. synthesized uniform $\mathrm{Ag}$ nanoparticles of $\sim 10 \mathrm{~nm}$ using aniline as the reducing agent and dodecylbenzenesulfonic acid (DBSA) as the stabilizer. ${ }^{61}$ It has been demonstrated that the formation of Ag nanoparticles was accomplished in a three-stage model, starting with mesoscopic structures, in which $\mathrm{Ag}$ ions were confined in the dispersed phases of DBSA and aniline, subsequently generating $\mathrm{Ag}_{2} \mathrm{O}$ nanocrystallites in situ upon the addition of excess $\mathrm{NaOH}$, followed by the reduction by aniline, leading to small uniform $\mathrm{Ag}$ nanoparticles.

2.2.2. Digestive ripening process in aqueous media. Digestive ripening is a post-synthetic process that enables the transformation of nanoparticles from polydisperse to monodisperse forms, leading to size homogeneity. Compared to the Ostwald ripening process, the digestive ripening process relies on ligand-mediated dissolution or etching of large nanoparticles and the growth of small nanoparticles at the expense of large ones (Fig. 4). ${ }^{62,63}$ A typical example of the digestive ripening process was demonstrated by Zhang et al. for the synthesis of hydrophilic monodisperse Ag nanoparticles of sizes 9-15 nm

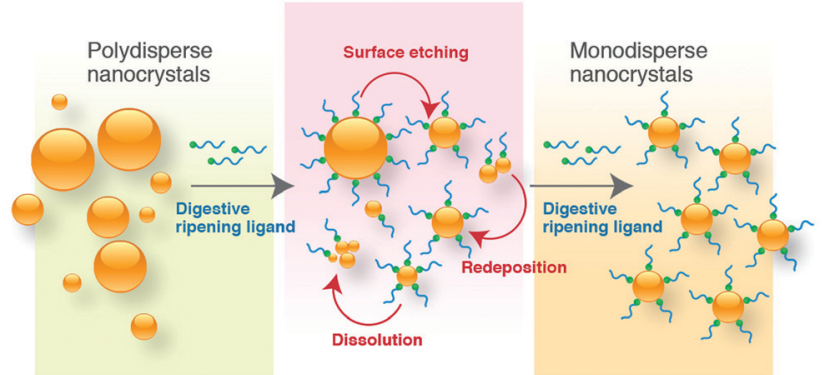

Fig. 4 Schematic illustration of the formation mechanism of uniform monodisperse nanocrystals by digestive ripening. with narrow size distributions. ${ }^{58}$ In this approach, the $\mathrm{Ag}$ nanospheres were synthesized in $N, N$-dimethylformamide (DMF) using chloride for the oxidative etching of $\mathrm{Ag}$ nanoparticles and diethylamine for surface stabilization, starting with the precursor of either an $\mathrm{Ag}^{+}$solution or an $\mathrm{AgCl}$ suspension. In the following study, Yu et al. synthesized hydrophilic monodisperse $\mathrm{Ag}$ nanoparticles with tunable sizes from $25 \mathrm{~nm}$ to $60 \mathrm{~nm}$ using oxygen-mediated digestive ripening process in the modified Lee-Meisel synthesis system. ${ }^{64}$ Unlike the conventional Lee-Meisel method, oxygen was introduced into the synthetic process and combined with nitrate/citrate ions to accomplish oxidative etching of the $\mathrm{Ag}$ nanoparticles. Therefore, the conversion of $\mathrm{Ag}$ nanoparticles from polydisperse to monodisperse form was achieved through the oxidative etching of the unstable triangular nanoplates, resulting in nearly spherical particles.

2.2.3. Seed-mediated growth method. Apart from the above modified strategies, the chemical reduction method can be optimized by introducing small Ag nanoparticles as seeds into the reaction system. The seed-mediated synthesis usually takes place in two steps-seed preparation and subsequent growth. The metal salts are firstly reduced with a strong reducing agent and successively added to a "growth" solution containing the metal precursor and a weak reducing agent as well as a shaping agent. As a result, the newly reduced metal can only assemble on the surface of the metal seeds and no nucleation of new particles occurs in the solution. Wan et al. modified the LeeMeisel method with seed-mediated growth to synthesize Ag quasi-spherical nanoparticles with tunable sizes up to $80 \mathrm{~nm} .^{65}$ The seed served as a nucleation center on which the Ag monomers formed from reduction, when added in the subsequent growth process. Hence, the ratio of the monomer to the seed plays an important role in controlling the size and shape of the Ag nanoparticles.

The seed-mediated growth method is also a common approach to prepare noble metal anisotropic nanostructures. Typically, a shaping agent is used in the solution to work as a suitable template that facilitates the growth process to yield shape-controlled nanostructures. For example, an early study was demonstrated by Jana et al. to prepare one-dimensional Ag nanostructures with a different aspect ratio via the seed-mediated growth approach. ${ }^{66}$ The growth of Ag nanorods and nanowires was achieved by the chemical reduction of $\mathrm{Ag}$ salt by ascorbic acid in the presence of the preformed $\mathrm{Ag}$ seed, cetyltrimethylammoniun bromide (CTAB), and $\mathrm{NaOH}$. Notably, the seed and the base concentration relative to the $\mathrm{Ag}^{+}$concentration were the key factors for producing large aspect ratio nanostructures. The seed-mediated growth approach was extended by Lee et al. to prepare Ag nanorods. ${ }^{67}$ It was reported that the preformed citrate-stabilized $\mathrm{Ag}$ seeds were added into the solution of Ag salt as well as ascorbic acid and CTAB. This work has demonstrated that the increase in the reaction temperature and $\mathrm{pH}$ led to a decrease in the aspect ratio of the $\mathrm{Ag}$ nanorods while improving the uniformity of sizes and shapes.

A recent work of Xia and co-workers demonstrated the preparation of $\mathrm{Ag}$ nanocubes in an aqueous system through 
A

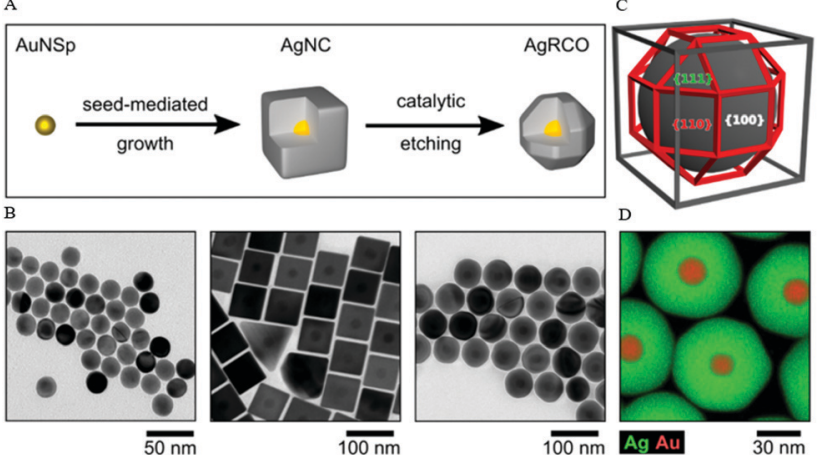

Fig. 5 (A) Schematic formation of Ag nanorhombicuboctahedra via seedmediated growth and $\mathrm{Cu}$-based etching process. (B) TEM images of the intermediate particles: $\mathrm{Au}$ nanoparticles, $\mathrm{Ag}$ nanocubes, and $\mathrm{Ag}$ nanorhombicuboctahedra. (C) 3-D sketch of a rhombicuboctahedron geometry. (D) EDX elemental distribution of $\mathrm{Ag}$ and $\mathrm{Au}$ in the final rhombicuboctahedrically-shaped nanoparticles. Reproduced from ref. 68 with permission from the American Chemical Society.

the formation of the $\mathrm{AgCl}$ octahedra by mixing $\mathrm{CF}_{3} \mathrm{COOAg}$ with cetyltrimethylammonium chloride (CTAC), which subsequently served as a source of elemental Ag. ${ }^{69}$ The nucleation and growth of $\mathrm{Ag}$ nanocubes was facilitated by photolysis in the presence of ascorbic acid and $\mathrm{FeCl}_{3}$. The $\mathrm{Fe}^{3+} / \mathrm{Fe}^{2+}$ redox pair acted as an oxidative etchant to selectively dissolve the twinned seeds while chloride ions acted as a specific capping agent toward the (100) facets, leading to the formation of singlecrystal Ag nanocubes with sharp corners and edges of an average edge length of 35-95 $\mathrm{nm}$. Lately, Steiner et al. developed a method to synthesize Ag nanoparticles with rhombicuboctahedral shape via the combination of seed-mediated growth and the Cu-based etching process. ${ }^{68}$ The formation of thermodynamically stable Ag rhombicuboctahedra was assisted by CTAC and the process began with the synthesis of single-crystalline spherical $\mathrm{Au}$ nanoparticles, followed by the induced epitaxial overgrowth of $\mathrm{Au}$ spheres, and led to $\mathrm{Ag}$ nanocubes of the same crystallinity. The Ag nanocubes were then selectively etched on isotropic plasmonic $\mathrm{Ag}$ nanorhombicuboctahedra using $\mathrm{Cu}\left(\mathrm{NO}_{3}\right)_{2}$ as the etching agent in order to overcome the limitation of low-index facets and to achieve a final spherical morphology (Fig. 5).

2.2.4. PVP-mediated polyol process in ethylene glycol (EG). Polyols such as EG in the chemical reaction usually act as both the solvent and the reducing agent; typical synthesis entails the reduction of the metal precursor by polyol in the presence of an appropriate capping agent at an elevated temperature. The capping agent PVP plays a remarkably important role in synthesizing Ag nanostructures with good stability and size/ shape uniformity through the interaction of the PVP chains onto the Ag surface, facilitated by the carbonyl oxygen and nitrogen atoms of the pyrrolidone unit. On the other hand, PVP has also been known as both the reductant and the stabilizer for the reduction of $\mathrm{Ag}$ salts by the hydroxyl end groups of PVP in the kinetically controlled growth of $\mathrm{Ag}$ triangular nanoplates. $^{70,71}$ Other examples of polyol synthesis include the size-controlled fabrication of $\mathrm{Ag}$ nanoparticles, ranging from 10 to $80 \mathrm{~nm}$ via a modified polyol method by controlling the ratio of PVP to $\mathrm{AgNO}_{3}$ in EG. ${ }^{72}$ To prepare $\mathrm{Ag}$ nanoparticles with size less than $50 \mathrm{~nm}$, ammonia solution was added to the $\mathrm{AgNO}_{3}$ solution as the complexing agent to form $\mathrm{Ag}\left(\mathrm{NH}_{3}\right)^{2+}$, which was more difficult to be reduced than $\mathrm{Ag}^{+}$. $\mathrm{Ag}$ grain growth was thus suppressed and the grains were coated with PVP, leading to smaller sizes of $\mathrm{Ag}$ nanoparticles. Lin et al. adopted the polyol synthesis of Ag nanowires using EG, PVP, and $\mathrm{AgNO}_{3}$ as the precursors. ${ }^{73}$ In this study, the growth characteristics of $\mathrm{Ag}$ nanowires were investigated by varying the synthesis temperature, concentration of $\mathrm{AgNO}_{3}$, and the rate at which $\mathrm{AgNO}_{3}$ was added. At lower concentrations of $\mathrm{AgNO}_{3}$, the diameter of the $\mathrm{Ag}$ nanowires decreased, hence leading to a higher aspect ratio. The result also suggested that high aspect ratios of $\mathrm{Ag}$ nanowires were obtained when the $\mathrm{PVP} / \mathrm{AgNO}_{3}$ molar concentration ratio was 16 .

Following the early studies of Xia and co-workers, a comprehensively study has been made to develop Ag nanostructures with well-controlled morphologies (i.e., nanocubes, nanorods, nanowires, and nanospheres) by fine tuning the ratio of $\mathrm{AgNO}_{3}$ and PVP in the polyol process. ${ }^{46}$ In the PVPmediated polyol process, the formation of a distinct morphology was determined by the crystallinity of the seeds through chemical interaction between the capping agent and various crystallographic planes of Ag and the extent of PVP coverage on the seed. By replacing the capping agent PVP with sodium citrate, the morphology of the Ag nanostructures was modified to thin triangular nanoplates and nanobelts due to the enlargement of the (111) rather than (100) planes. The polyol synthesis of controlling the specific seed structure to grow $\mathrm{Ag}$ nanostructures was later extended to selectively produce Ag pentagonal nanowires, cuboctahedra, nanocubes, nanobars, bipyramids, and nanobeams. ${ }^{74,75}$ These shape-defined $\mathrm{Ag}$ nanostructures were synthesized firstly through the reduction of $\mathrm{Ag}^{+}$ions by EG. The reduced $\mathrm{Ag}$ atoms subsequently agglomerated to form nuclei of fluctuating structures, which are greatly dependent on their size and the thermal energy available. In many cases, the nuclei incorporated twin boundary defects because such defects enabled a lower surface energy. As the nuclei grew, the fluctuations stabilized and they were fixed as a multiply twinned, singly twinned, or single-crystal seed. Eventually, the crystallinity of the seeds governed the nanostructures of a specific shape.

Considering the long reaction time for the PVP-mediated polyol synthesis, the incorporation of a trace amount of sulfide anions (i.e., $\mathrm{Na}_{2} \mathrm{~S}$ or NaHS) was reported to accelerate the production rate of $\mathrm{Ag}$ nanocubes due to an increase in the reduction rate of $\mathrm{Ag}$ ions. ${ }^{76}$ When compared to the conventional polyol process, the reaction time was significantly shortened from $16-26 \mathrm{~h}$ to $3-8 \mathrm{~min}$. At this enhanced rate, the evolution of $\mathrm{Ag}$ nanocubes was dominated by the fast kinetic growth of single-crystal seeds. This sulfide-assisted polyol synthesis is useful for the preparation of $\mathrm{Ag}$ nanocubes to study the optical properties in the single isolated nanoparticles. ${ }^{77}$ Besides the conventional polyol process, a recent report of PVP-mediated polyol synthesis has also shown that the controlled growth of $\mathrm{Ag}$ nanoparticles could be performed in polyol solvent mixing with a co-solvent (i.e., propylene carbonate). ${ }^{78}$ This is to prevent 
heterogeneous nucleation when anionic additives or impurities are present in the reaction mixture; in particular, the formation of $\mathrm{AgCl}$ can occur when chloride is present and the surface of $\mathrm{AgCl}$ can serve as a nucleation site for $\mathrm{Ag}(0)$. Contrary to conventional polyol solvents such as EG and propylene glycol, the solubility of $\mathrm{AgCl}$ in the mixed solvent is sufficiently high such that heterogeneous nucleation on $\mathrm{AgCl}$ can be prevented.

2.2.5. PVP-mediated synthesis in DMF. In PVP-mediated synthesis, the choice of solvent is important and DMF can also be used as a solvent besides EG. The noteworthy difference between the two solvent/reducing agent is that the preferential facets of $\mathrm{Ag}$ nanostructures formed from the reduction of $\mathrm{AgNO}_{3}$ in EG and DMF are different. For instance, the adsorption of PVP to (100) Ag facets dominates in EG, whereas PVP interacts differently in DMF and stabilizes (111) facets relative to the (100) facets. DMF has been known as a powerful reducing agent against $\mathrm{Ag}^{+}$ions and the modulation of the surface energy of $\mathrm{Ag}$ seeds by PVP in DMF is different from EG. ${ }^{79-81}$ Hence, $\mathrm{Ag}$ nanostructures having (100) facets such as cubes, righttriangular bipyramids, pentagonal rods, and wires are dominantly produced in EG, while nanostructures having (111) facets such as triangular and hexagonal plates, decahedra, and icosahedra are preferably produced in DMF.

Pastoriza-Santos et al. generated Ag nanoprisms in DMF with the assistance of PVP as a stabilizer. The size of the nanoprisms was tuned by the reaction time at reflux. However, this method produced a mixture of nanoprisms and nanospheroids during the synthetic process and the latter has to be removed through centrifugation. ${ }^{82}$ Gao et al. synthesized nanostructured Ag decahedrons via the PVP-mediated DMF reduction of $\mathrm{AgNO}_{3} .{ }^{83}$ This study revealed that $\mathrm{Ag}$ decahedrons capped by PVP contained five-twinning structures with distinct twinning boundaries, likely formed through the assembling of five tetrahedrons. An important suggestion in the present study is that the preferential facets of nanostructured Ag synthesized in DMF could be altered from the (100)-type to the (111)-type. The PVP-mediated DMF reduction process was extended by Tsuji et al. to prepare decahedral and icosahedral nanostructures as well as a series of their intermediate particles, which consist of a combination of two and more tetrahedra. ${ }^{84}$ It was proposed that the Ag polyhedra was synthesized via the stepwise growth of tetrahedral units on a specific (111) facet of intermediate species. In other words, the shape selective formation of decahedron and icosahedron was dependent on the concentration of PVP, which yielded a different extent of PVP coverage of each (111) facet. Later, the work was continued with PVP-assisted DMF reduction to produce nanoflag-type Ag nanostructures from nanorod seeds. ${ }^{79}$ These nanostructures were formed via a two-step process with EG and DMF as the reducing agent and solvent, starting with the synthesis of pentagonally twinned nanorods by the polyol reduction of $\mathrm{AgNO}_{3}$ in EG and PVP. In the second step, $\mathrm{AgNO}_{3}$ reduction took place in DMF with Ag nanorod seeds and PVP. The resulting products obtained were nanoflag-type structures having (111) facets. The shape evolution of these Ag nanostructures from trapezoid and triangular plates to tetrahedral structures was made possible by tuning the ratio of $\mathrm{AgNO}_{3}$ of the second step relative to the first step. The driving force of significant shape evolution was attributed to the changes in the preferential adsorption of PVP from (100) Ag facets in EG to (111) Ag facets in DMF.

\subsection{Gold nanostructures}

Traditionally, poorly reactive or chemically inert bulk Au is considered as a safe material for biomedical applications. To date, $\mathrm{Au}$ is one of the most studied materials owing to its unique chemical, electronic, and optical properties when the feature size decreases to the nanometer scale. A large number of studies are dedicated to the development of nanostructured Au with numerous morphologies to exploit the innovative nanoscale chemical effects for diverse applications such as biomedical, catalysis, energy, and environmental technologies. ${ }^{85-87}$

2.3.1. Seed-mediated growth method. The seed-mediated growth method has been commonly used to synthesize almost all the possible fundamental shapes of faceted Au nanocrystals. For instance, Au nanocrystals encased with different proportions of the three low-index facets such as (100), (111), and (110) could be readily obtained in both single-crystal structures (cubes, octahedrons, cuboctahedrons, tetrahedrons, rhombic dodecahedrons, and rods) and twinned structures (decahedra, icosahedra, wires, and prisms). Also, the $\mathrm{Au}$ nanocrystals encased by high-index facets include trisoctahedrons with (221) facets, tetrahexahedrons with (037) facets, truncated ditetragonal prisms with (310) facets, concave cubes with (720) facets, and hexoctahedrons with (321) facets. ${ }^{8-90}$

The pioneering study of seed-mediated method was developed by Murphy and co-workers ${ }^{91}$ and later modified by El-Sayed and Nikoobakht. ${ }^{92}$ The work of Murphy demonstrated the preparation of $\mathrm{Au}$ nanorods using citrate-capped $\mathrm{Au}$ seeds synthesized from the reduction of $\mathrm{HAuCl}_{4}$ with borohydride. The ratio of the seed to the metal salt was then used to control the aspect ratio of the $\mathrm{Au}$ nanorods. ${ }^{91,93}$ The seed catalyzed the reduction of $\mathrm{Au}^{\mathrm{I}}$ to $\mathrm{Au}^{0}$ when added to $\mathrm{HAuCl}_{2}$ solution, which was previously prepared from $\mathrm{HAuCl}_{4}$ and ascorbic acid in the presence of $\mathrm{CTAB}$ and $\mathrm{AgNO}_{3} \cdot{ }^{94}$ The role of $\mathrm{Ag}^{+}$metal ions was significant for nanorod growth; in particular, the presence of metal ions increased the yield of Au nanorods from $20-40 \%$ to nearly $100 \%$. On the other hand, the high yield of Au nanorods in the presence of $\mathrm{Ag}$ ions was also accompanied with a lower aspect ratio. The essential role of $\mathrm{Ag}^{+}$was also demonstrated by Zhang et al. to synthesize concave nanocubes, enclosed by 24 high-index (720) facets via a modified seed-mediated growth method. ${ }^{95}$ Another key component in the synthesis was the chloride-containing surfactant that determined the product morphology. Specifically, this protocol has been modified from the usual bromide- to chloride-containing surfactant. Therefore, the use of the chloride counterion in the surfactant and combination with $\mathrm{Ag}^{+}$resulted in the formation of concave cubes (i.e., CTAC) rather than a convex cubic structure (i.e., CTAB).

Likewise, the effect of halide ions during the seed-mediated growth has been demonstrated to prepare three different $\mathrm{Au}$ 
nanostructures (i.e., nanoprism, nanorod, and nanorice) by controlling the content of halide ions. ${ }^{96}$ It was reported that the addition of a small amount of iodide ions in the growth solution induced a change in the shape from $\mathrm{Au}$ nanorods to triangular nanoprisms. The presence of the iodide ions suppressed the overall crystal growth through selective binding on the $\mathrm{Au}(111)$ surface, resulting in the formation of $\mathrm{Au}(111)$ faced triangular nanoprisms. On the contrary, a drastic change in the morphology from nanorod to nanorice was observed when the counterion of the surfactant was replaced with chloride ions (i.e., CTAB to CTAC). This study clearly demonstrated the influence of halide ion adsorption in the morphological transformation of $\mathrm{Au}$ nanostructures. Similarly, Chen et al. synthesized the triangular $\mathrm{Au}$ nanoplates through the iodideassisted approach. ${ }^{97}$ The role of iodide facilitated the formation of Au nanoplates by selective binding onto the $\mathrm{Au}(111)$ facets and oxidative etching by tri-iodide ions to eliminate other shaped impurities, leaving behind dominant $\mathrm{Au}$ nuclei with planartwined structures. Moreover, the edge length of triangular $\mathrm{Au}$ nanoplates were tunable $(\sim 40$ to $\sim 120 \mathrm{~nm})$ by varying the concentration of the reagents such as ascorbic acid, KI, and $\mathrm{NaOH}$, while keeping the concentrations of CTAC and $\mathrm{Au}^{3+}$ constant.

A comprehensive study on the effect of halide ions (chloride, bromide, and iodide) in the seed-mediated synthesis of $\mathrm{Au}$ anisotropic nanostructures was reported by Langille (Fig. 6). ${ }^{88}$ This comprised of two different sets of particle shapes through kinetic control, surface passivation, or a combination of both, either with or without Ag ions. Specifically, without Ag ions, the rate of reaction was the leading factor that governed the product growth. Slower rates of reaction yielded lower-energy surface facets and could be achieved by adding a lower amount of ascorbic acid or a larger halide (i.e., bromide or iodide). On the other hand, $\mathrm{Ag}$ ions could be used as an underpotential deposition agent to control the particle growth through surface

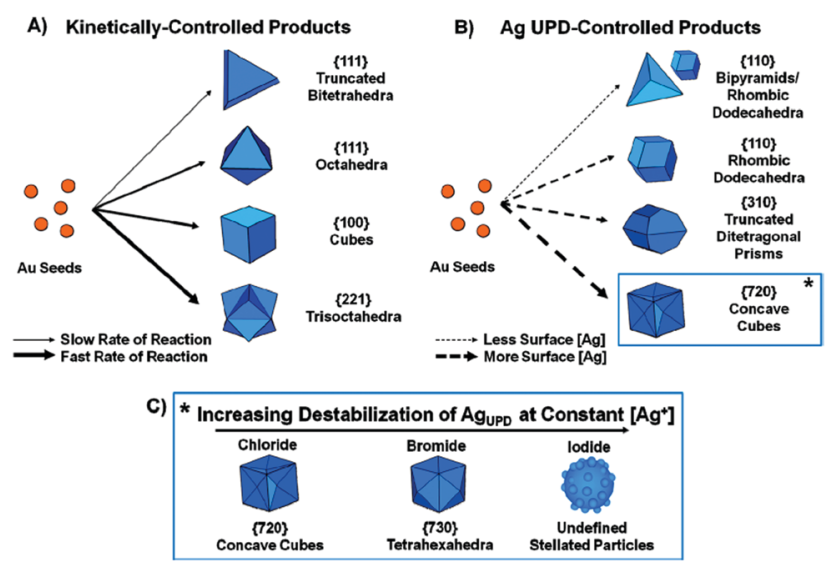

Fig. 6 Schematic illustration of the seed-mediated synthesis of Au nancrystals through the use of halides and Ag ions. (A) Kinetically controlled products in the absence of $\mathrm{Ag}$ ions. (B) Ag underpotential depositioncontrolled products. (C) High-index faceted products in the presence of halides and Ag ions. Reproduced from ref. 88 with permission from the American Chemical Society. passivation. Higher amounts of $\mathrm{Ag}$ on the particle surface yielded higher-index surfaces. Huang and co-workers reported a seed-mediated protocol for the preparation of Au nanostructures with systematic morphological evolution from truncated cubic to cubic, trisoctahedral, and rhombic dodecahedral structures. ${ }^{98}$ Shape transformation was achieved by adjusting the volume of ascorbic acid added into the growth solution to control the rate of $\mathrm{Au}$ atom addition on the crystal surface, leading to the formation of Au nanostructures. In this study, the use of CTAC surfactant and a tiny amount of $\mathrm{NaBr}$ into the growth solution was found to be critical to this morphological evolution. Later, the effect of bromide ions was replaced with iodide ions to induce the morphological evolution from rhombic dodecahedral to octahedral structures. ${ }^{99,100}$ Compared to chloride and bromide ions, iodide ions with a low reduction potential could act as a reducing agent in the presence of $\mathrm{AuCl}_{4}$ species to modulate the reduction rate along different crystallographic directions, resulting in the morphological evolution. Another work that explored the growth of $\mathrm{Au}$ nanocrystals with systematic shape evolution was studied by Kuo et al. ${ }^{101}$ Using seed-mediated method to grow Au nanocubes, rhombic dodecahedra, and octahedra as the starting points, shape transformation from rhombic dodecahedral to trisoctahedral and cubic structures could be achieved by adjusting the volume of $\mathrm{HAuCl}_{4}$ solution as well as incorporating $\mathrm{NaBr}$ in the process. On the other hand, the shape transformation from octahedral to corner-truncated octahedra, edge- and corner-truncated octahedra, and rhombic dodecahedra could be achieved in the presence of KI. The next examples describe the preparation of Au nanocrystals with unique shapes such as tetrahedral and trapezohedral through a seed-mediated growth process. Xia and co-workers reported the formation of $\mathrm{Au}$ tetrahedra with tunable sizes from 30 to $60 \mathrm{~nm} .{ }^{89}$ The synthesis involved the use of single-crystal and spherical seeds in the presence of CTAB/CTAC as well as the manipulation of the reaction kinetics to promote an unsymmetrical growth pattern for the seeds, leading to the formation of tetrahedral products. On the hand, Niu et al. synthesized the trapezohedral $\mathrm{Au}$ nanocrystals by employing dimethyl sulfoxide (DMSO) as the solvent for shaping Au nanocrystals with (311) crystal facets. ${ }^{102}$ The success of this synthesis relied on the ambidentate nature of the DMSO solvent, where the lone-pair of electrons on the oxygen and sulphur atoms could coordinate to the Au surface, thus stabilizing the high-index (311) facets through a "twocenter bonding"' mode.

In addition to seed-mediated synthesis, a purification protocol was developed by Scarabelli et al. to achieve shape monodispersity. ${ }^{103}$ This synthetic scheme for the preparation of monodisperse $\mathrm{Au}$ nanotriangles with the size of the edge length ranging from 50 to $150 \mathrm{~nm}$ was comprised of three consecutive steps: (1) generation of CTAC-coated Au seeds, (2) fast addition of the generated seeds to a final growth solution, and (3) purification of the products. Purification was carried out by flocculation and subsequent precipitation by CTAC micelles to remove the by-product particles. The high uniformity enabled the formation of the self-assembly and closely-packed 
$\mathrm{Au}$ nanotriangle monolayers. Apart from the Au nanostructures with well-controlled morphologies described above, it is worth mentioning that the seed-mediated method can also be extended to synthesize anisotropic Au nanocrystals with high chiroptical activity through the templating effect of chiral micelles formed in the presence of dissymmetric co-surfactants. ${ }^{104}$ This work was demonstrated by González-Rubio and co-workers to synthesize $\mathrm{Au}$ nanorods, employing 1,1'-bi(2-naphthol) as a cosurfactant with axial chirality to induce the assembly of surfactants into helical micelles, which tend to coil around the Au nanorods, leading to products with chiral features. Compared to the conventional nanorods, this surfactant-assisted seeded growth of Au nanorods possess chiral features and the chiral plasmonic nanostructures are promising candidates for real-life applications owing to their significantly larger optical chirality than that of natural chiral materials. ${ }^{105,106}$

2.3.2. Polyol synthesis. Polyol approach is a straightforward and effective approach for the controllable synthesis of $\mathrm{Au}$ nanostructures. Seo et al. synthesized a series of $\mathrm{Au}$ nanostructures (i.e., octahedra, truncated octahedra, cuboctahedra, cubes, and higher polygons) by increasing the concentration ratio of $\mathrm{Ag}$ to $\mathrm{Au}$ precursors while refluxing in 1,5-pentanediol. ${ }^{107}$ The concentration of $\mathrm{Ag}$ was the key factor in the reaction for structural fine-tuning by the selective growth of (111) and/or the restriction of (100). For instance, when the Ag concentration was high and exceeded the selective deposition condition, seed growth was completely inhibited along all the directions, resulting in smaller spherical particles (higher polygons). Besides the metal ions, the cationic surfactant poly(diallyldimethylammonium) chloride (PDDA) has been used as an effective capping agent to synthesize $\mathrm{Au}$ nanostructures in the polyol process owing to the excellent adsorption abilities on the particular crystal planes. The PDDA-mediated polyol route was used to develop Au octahedra nanostructures in EG or 1,5pentanediol solution. It was found that the formation of octahedral $\mathrm{Au}$ nanostructures was due to the selective adsorption of surfactant PDDA molecules on the (111) planes of the Au nuclei, which restricted the growth rate along the (111) direction. ${ }^{108,109}$ Zhang et al. synthesized the high-index facets icosidodecahedral $\mathrm{Au}$ nanostructures by the PDDA-mediated polyol route. ${ }^{110}$ By adjusting the reaction temperature, time, and concentration of PDDA, the icosidodecahedral Au nanostructures with unique hyperpolyhedral shapes having surfaces enclosed by 8 low-index (111) facets and 24 high-index (310) facets were formed without the use of foreign metal ions or seeds in the reaction.

2.3.3. Template-directed approach. In addition to the faceted geometries (i.e., cubes, octahedral, and dodecahedra) as well as the one-dimensional structures (i.e., rods and wires) that have been discussed above, there is another class of Au nanostructures with unique morphology, which is the branched architecture (i.e., urchins, ${ }^{111}$ stars, ${ }^{112,113}$ crosses, ${ }^{114,115}$ and multipods $\left.{ }^{116,117}\right)$. Most of the branched $\mathrm{Au}$ nanostructures can be achieved through the seed-mediated method, as discussed preciously. ${ }^{86}$ There are also other synthetic protocols that have been employed to produce the branched $\mathrm{Au}$ nanostructures. For instance, Xia and co-workers introduced a template-directed

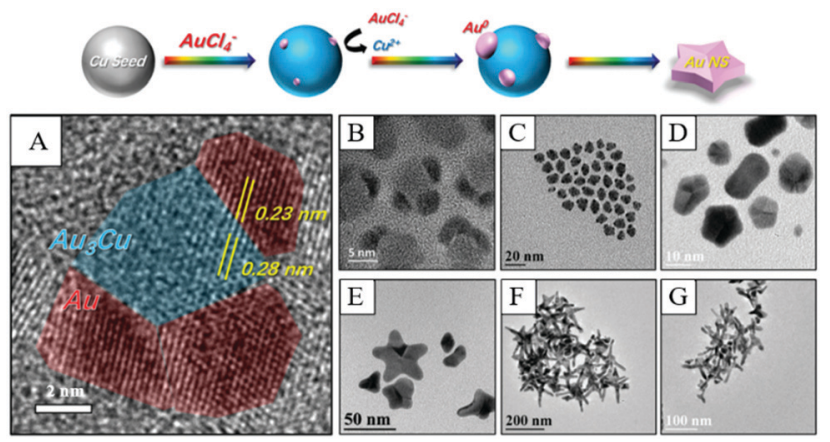

Fig. 7 Schematic fabrication of $\mathrm{Cu}$-induced formation of Au nanostars. (A) HRTEM image of nucleates from panel (B). (B)-(G) TEM images of nanocrystals obtained at different reaction times after the injection of the Au precursor. Reproduced from ref. 119 with permission from the Royal Society of Chemistry.

approach for synthesizing Au multipods. During this process, a galvanic replacement reaction took place between the threedimensional (3-D) porous lattice Fe template and the $\mathrm{Au}^{+}$precursor, which led to the formation of highly branched $\mathrm{Au}$ nanostructures in the void space of the lattice. In another example, our research group has prepared the cross-shaped Au nanostructures through the chemical reduction of $\mathrm{AuCl}_{3}$ using $\mathrm{Cu}$-based reductants in the presence of oleylamine. ${ }^{114,115,118}$ In this method, the $\mathrm{Cu}$-induced formation of the $\mathrm{Au}$ nanocrosses was highly dependent on the selection of the $\mathrm{Cu}$ species in the reaction such as $\mathrm{Cu}^{+}$, $\mathrm{Cu}$ nanostructures, or their combination, which controlled the reduction rate and led to $\mathrm{Au}$ nanocrosses with tunable size. In a recent study, Zhu et al. synthesized the multi-branched Au nanostructures (i.e., nanostars) through the galvanic replacement of $\mathrm{Cu}$ by $\mathrm{Au}$ in the organic media. ${ }^{119}$ This synthesis was based on the in situ formation of $\mathrm{Cu}$ nanoparticle seeds assisted by oleylamine, followed by galvanic exchange of $\mathrm{Cu}$ by $\mathrm{Au}$, which initiated the growth of branched structures (Fig. 7). In this study, Cu(I)-complex was found to be an essential component for generating the multi-branched architectures. By extending the reaction time, a longer branch length was achieved, thus resulting in branched Au nanocrystals with tunable armlength (50-200 nm).

\subsection{Bimetallic metal nanostructures}

Significant progress has been made on the controlled synthesis of single-metal nanostructures (as discussed in the previous section). On the contrary, the fabrication of bimetallic nanostructures consisting of $\mathrm{Cu}, \mathrm{Ag}$, and $\mathrm{Au}$ with controlled sizes/ shapes has been less extensively studied and the strategies reported so far are relatively limited. The preparation methods using the solution-based approaches reported thus far are summarized in the following section.

2.4.1. Co-reduction method in aqueous media. From the literature, the synthesis of bimetallic nanostructures can be readily carried out through the simple chemical reduction of the salt mixture with reducing agents such as sodium borohydride, hydrazine, and hydrogen. ${ }^{120-123}$ Generally, during the co-reduction process, the highest redox potential metal species 
precipitates first, forming a core on which the second component is deposited as a shell. ${ }^{120}$ The sequence of deposition can be altered if the metal with the higher redox potential is bound strongly with a ligand, yielding an inverse core-shell arrangement. In an early study of Ag-Pd nanoparticles $(70: 30$ weight\%), the co-reduction of $\mathrm{Ag}$ and $\mathrm{Pd}$ led to the formation of the Pd core-Ag shell clusters due to the higher redox potential of Pd. ${ }^{120}$ Conversely, co-precipitation in the ammonia solution containing the two metal salts and hydrazine led to the formation of the reverse $\mathrm{Ag}$ core-Pd shell cluster because of the stronger binding of $\mathrm{NH}_{3}$ to $\mathrm{Pd}$ than to Ag. In an example of $\mathrm{Au}-\mathrm{Ag}$ core-shell nanocrystal synthesis, rhombic dodecahedral $\mathrm{Au}$ nanocrystals were used as the cores to produce the coreshell nanocrystals with cubic, truncated cubic, cuboctahedral, truncated octahedral, and octahedral structures. ${ }^{124}$ In this core-shell growth strategy, Au nanocrystals were added to an aqueous mixture of the CTAC surfactant, $\mathrm{AgNO}_{3}$, ascorbic acid, and $\mathrm{NaOH}$ to obtain the products. By adjusting the reaction temperature, the octahedra were obtained at a higher growth rate, while slower growth promoted cube formation.

In recent years, the green synthesis of nanoparticles has emerged as an attractive alternative route to traditional synthetic methods due to their potential in minimizing the toxicity of the nanoparticles. Specifically, there are three key aspects to consider with regard to green synthesis: solvent medium, nontoxic reducing agents, and non-hazardous stabilizing agents. The commonly used green sources include bacteria, fungi, actinomycetes, yeasts, viruses, polysaccharides, and plant extracts (leaves, flower, stem bark, fruit peels, seed, etc.), which have been reported to synthesize a series of metal nanoparticles. ${ }^{125-127}$ Among these, plant-mediated synthesis of metal nanoparticles appears to be a more favorable way because plant-modified materials are easy and inexpensive to be cultured as compared to those of microorganisms. Moreover, the eco-friendly synthetic approach is relatively simple, rapid, and inexpensive, which can be easily scaled up for larger production due to uncomplicated reaction procedures, which is typically done in an aqueous solution under ambient conditions.

Plant extracts contain a number of secondary metabolites and biomolecules in the form of coenzymes, which are vitaminbased intermediates that enable the reduction of metal ions to nanoparticles in a single step reduction process. ${ }^{128}$ In addition, these metabolites can have a dual role as reducing and stabilizing agents to prevent the aggregation and agglomeration of the nanoparticles during synthesis. The formation of metallic nanoparticles through biosynthesis is influenced by the type of plant extract, concentration (plant extract and metal salt), as well as the temperature and $\mathrm{pH}$, which control the shape and size of the synthesized nanoparticles. ${ }^{129-131}$ Our group reported the flower-shaped $\mathrm{Au}$ nanostructures through aggregation-induced mechanism using star fruit (Averrhoa carambola) juice extract. The fruit extract is rich in in vitamin $\mathrm{C}$ and polyphenolic antioxidants, which can act as both reducing and stabilizing agents to synthesize branched gold nanoflowers, without additional shape-directing agents. ${ }^{132,133}$ Likewise, Nguyen et al. reported the biosynthesis of Ag nanostructures using three kinds of leaf extracts (Phyllanthus urinaria, Pouzolzia zeylanica, and Scoparia dulcis). When using Pouzolzia zeylanica leaf extracts as the reducing agents and stabilizers, diversified morphology was obtained, while the others showed a spherical morphology. To prepare $\mathrm{Au}-\mathrm{Ag}$ bimetallic nanoparticles, various plant extracts have been reported as effective reducing agents and stabilizers, including Anacardium occidentale, ${ }^{134}$ Gloriosa superba,${ }^{135}$ Solidago canadensis, ${ }^{136}$ Commelina nudiflora, ${ }^{137}$ Pulicaria undulata,${ }^{138}$ and Muntingia calabura ${ }^{139}$ In one example, Sheny et al. revealed that the biomolecules responsible for the reduction and stabilization of $\mathrm{Au}$ and $\mathrm{Ag}$ nanoparticles are found to be different. The proteins and polyols present in cashew leaves (Anacardium occidentale) are mostly responsible for the reduction. ${ }^{134}$ Both the formation of $\mathrm{Au}-\mathrm{Ag}$ core-shell nanoparticles $(6.5 \mathrm{~nm})$ and $\mathrm{Au}-\mathrm{Ag}$ alloy nanoparticles $(8 \mathrm{~nm})$ are in agreement with the UV-vis spectrum, featuring two plasmon bands and a single SPR band, respectively. Sharma et al. demonstrated the biosynthesis of Au-Ag bimetallic nanoparticles using clove bud extract. ${ }^{140}$ During the biosynthesis, the metal salt solutions containing $\mathrm{Au}(\mathrm{III})$ and $\mathrm{Ag}(\mathrm{I})$ are co-reduced competitively to form the $\mathrm{Au}-\mathrm{Ag}$ alloy nanoparticles in the presence of the clove bud extract. The formation of hexagonal and polygonal shaped $\mathrm{Au}-\mathrm{Ag}$ alloy nanoparticles with an average particle size of $16 \mathrm{~nm}$ was achieved through flavonoids and eugenol present in the extract. Alti $e t$ al. synthesized three kinds of $\mathrm{Au}-\mathrm{Ag}$ bimetallic nanoparticles (10-12 nm) using fenugreek, coriander, and soybean leaf extracts. ${ }^{131}$ The biosynthesized $\mathrm{Au}-\mathrm{Ag}$ bimetallic nanoparticles are in the form of an alloy and not a mixture of individual metal particles, as evident from the UV-vis spectrum.

2.4.2. Co-reduction method in organic media. Recently, multiple noble metal nanoparticles with core-shell construction were synthesized by co-reduction at elevated temperatures using different metal precursors in oleylamine. ${ }^{141}$ In this hightemperature reduction reaction, oleylamine acted as both the reducing and stabilizing agent to develop the one-step formation of core-shell structured nanoparticles. The strategy in this work utilized the difference in the reduction kinetics of various noble metal precursors in oleylamine to develop single or multiple shells structured nanoparticles with Ag or Au residing in the core regions. Moreover, the core-shell nanoparticles with the Ag core could be altered into hollow or cage-bell structure. The coreduction of metal precursors was also demonstrated to produce bimetallic $\mathrm{Cu}-\mathrm{Au}$ nanoparticles with different compositions (i.e., $\mathrm{CuAu}_{3}, \mathrm{CuAu}$, and $\mathrm{Cu}_{3} \mathrm{Au}$ ) in organic solvent at $280{ }^{\circ} \mathrm{C} .{ }^{142}$ In this high-temperature reduction reaction, oleylamine acted as both the reducing and stabilizing agent to develop bimetallic nanoparticles with the average size close to $10 \mathrm{~nm}$ and displayed significant stability against oxidation in the ambient atmosphere. Importantly, the synthetic method facilitated the assembly of uniform $\mathrm{Cu}-\mathrm{Au}$ bimetallic nanoparticles into ordered monolayers for further catalytic activity study.

Besides, the high-temperature reduction method can be used to prepare monodisperse $\mathrm{Au}-\mathrm{Ag}$ alloy nanoparticles at $150{ }^{\circ} \mathrm{C}$ in organic solvent, with controlled diameter from 6 to $13 \mathrm{~nm} .{ }^{143}$ In this method, the key is to control the surface 
plasmon resonance (SPR) frequency and the size of the $\mathrm{Au}-\mathrm{Ag}$ alloy nanoparticles not only by varying the precursor ratio but also by varying the surfactant composition (i.e., oleylamine, dodecanethiol, and 1-hexadecylamine). The high-temperature reduction method was also used to successfully synthesize $\mathrm{Cu}-\mathrm{Ag}$ bimetallic nanoparticles in the presence of trioctylphosphine and oleylamine.${ }^{15}$ In a modification of the co-reduction method, Chen et al. reported a seed-based diffusion protocol driven by the difference in the redox potentials between $\mathrm{Cu}$ and $\mathrm{Au}$ metals for generating intermetallic $\mathrm{Cu}-\mathrm{Au}$ nanoparticles. ${ }^{144}$ In the presence of oleic acid and tri- $n$-octylamine, $\mathrm{Cu}^{2+}$ ions were reduced to $\mathrm{Cu}$ atoms or clusters and subsequent collided with the active sites of preformed Au seeds, leading to the diffusion of active $\mathrm{Cu}$ atoms into the Au crystal lattice to form the $\mathrm{Cu}-\mathrm{Au}$ alloy. In this approach, the size control of the $\mathrm{Cu}-\mathrm{Au}$ nanoparticles could be fine-tuned by the selection of Au seeds while the selective synthesis of intermetallic $\mathrm{Cu}-\mathrm{Au}$ and $\mathrm{Cu}_{3} \mathrm{Au}$ nanoparticles could be achieved by changing the molar ratio of the precursors.

2.4.3. Galvanic replacement reaction. The galvanic replacement reaction is a versatile strategy for engineering the architectural metal nanostructures with hollow interiors and porous walls. During the redox process, the oxidized metal acts as a sacrificial template for the formation of hollow metal nanostructures through galvanic exchange. ${ }^{145-147}$ Several Au-based hollow nanostructures with different morphologies (i.e., cubic nanoboxes and cubic nanocages) have been produced using this strategy. ${ }^{11,148}$ These hollow nanostructures are attributed to the template-engaged hollowing-out mechanism. ${ }^{149}$ Accordingly, the electrochemical potential of $\mathrm{Au}$ is more positive than that of $\mathrm{Ag}$ (i.e., the reduction potential of $\mathrm{AuCl}_{4}{ }^{-} / \mathrm{Au}$ is $0.99 \mathrm{~V} v$ s. SHE and $\mathrm{AgCl} / \mathrm{Ag}$ is $0.22 \mathrm{~V} v$ s. SHE); $\mathrm{Ag}$ nanocubes will act as the sacrificial template/reducing agent while $\mathrm{Au}^{3+}$ will act as the oxidizing agent. ${ }^{148,150}$ As the reaction proceeds, the electrons generated in the oxidation process migrate to the $\mathrm{Ag}$ nanocube surface and reduce $\mathrm{Au}^{3+}$ to $\mathrm{Au}^{0}$. The $\mathrm{Au}$ atoms grow epitaxially over the Ag nanocubes because both $\mathrm{Au}$ and $\mathrm{Ag}$ possess the same face-centered cubic structure with closely matched lattice constants. The reaction begins with tiny voids on the surface of the nanocube, allowing ionic species to continuously diffuse in and out of the oxidation site. Eventually, the void becomes larger, forming a deep hole that gradually turns into a seamless nanobox composed of the $\mathrm{Au}-\mathrm{Ag}$ alloy. When more $\mathrm{Au}^{3+}$ are added, further etching process of the nanobox takes place, which leads to the formation of Au-based porous nanocages. Puntes and co-workers reported the preparation of $\mathrm{Au}-\mathrm{Ag}$ double-walled nanoboxes through the simultaneous action of galvanic replacement and the Kirkendall effect (Fig. 8). ${ }^{151}$ In this method, Ag was used as the template, PVP as the surfactant, $\mathrm{CTAB}$ as the surfactant and complexing agent, $\mathrm{Au}$ as the oxidizing agent, and ascorbic acid as the reducing agent to facilitate the morphological evolution from $\mathrm{Ag}$ nanocubes to a series of $\mathrm{Au}-\mathrm{Ag}$ hollow nanostructures with various morphologies and compositions at room temperature.

Likewise, different Ag nanostructures have been adopted as templates to tune the morphology and shape of $\mathrm{Au}-\mathrm{Ag}$

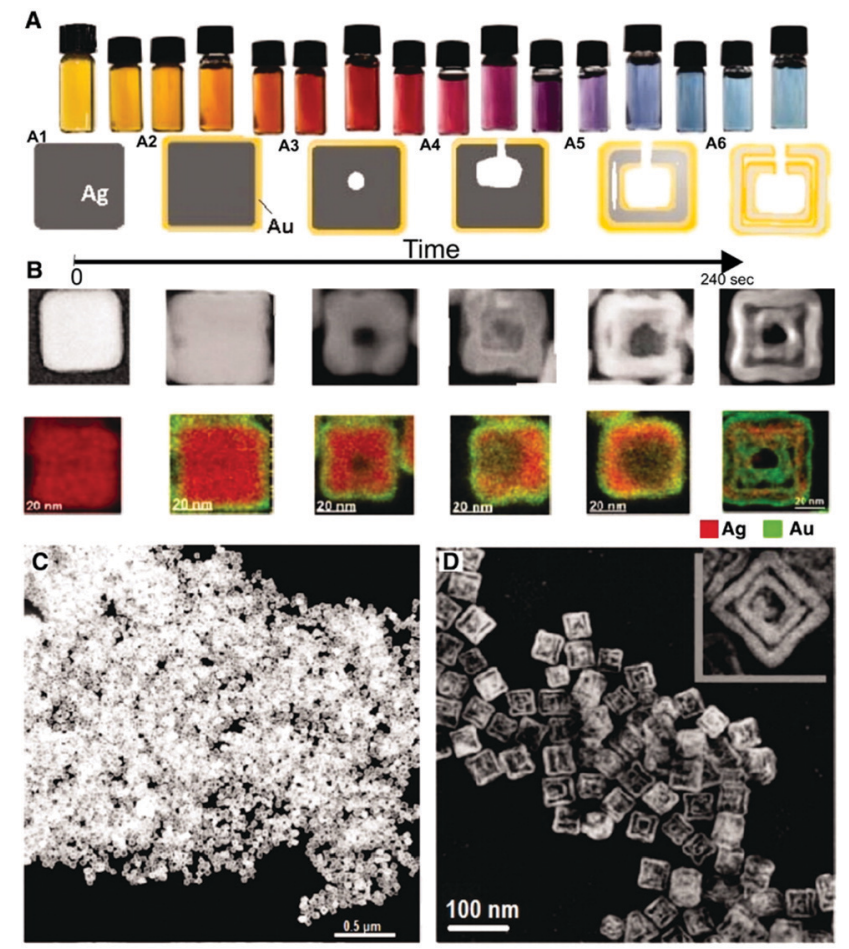

Fig. 8 Preparation of $\mathrm{Au}-\mathrm{Ag}$ double-walled nanoboxes from $\mathrm{Ag}$ nanocubes. (A) Optical images and schematic illustration of the morphological evolution of $\mathrm{Au}-\mathrm{Ag}$ double-walled nanoboxes through simultaneous galvanic replacement and Kirkendall effect. (B) Corresponding highangle annular dark field STEM images and EDX mapping. (C and D) TEM images of Au-Ag double-walled nanoboxes. Reproduced from ref. 151 With permission from the American Association for the Advancement of Science.

nanostructures by means of galvanic replacement. For example, twined Ag nanoparticles and single-crystalline Ag nanowires were used as the template to produce $\mathrm{Au}-\mathrm{Ag}$ alloy rings and Ag@AuAg core-shell wires. ${ }^{152} \mathrm{Ag}$ nanospheres were employed as the template for the synthesis of $\mathrm{Au}-\mathrm{Ag}$ nanorings in the presence of $\mathrm{AuCl}_{4}{ }^{-}$and PVP aqueous solution. ${ }^{153}$ In another example of the $\mathrm{Au}-\mathrm{Ag}$ nanoalloy, Camargo and co-workers synthesized $\mathrm{Au}-\mathrm{Ag}$ nanodendrites through a combination of galvanic replacement and precursor reduction, employing Ag nanoparticles as seeds, hydroquinone as the reducing agent, and PVP as the stabilizer. ${ }^{154}$ The size and surface morphology of $\mathrm{Au}-\mathrm{Ag}$ nanodendrites were tunable in the range of $45-$ $148 \mathrm{~nm}$ by varying the concentration of the $\mathrm{Ag}$ nanoparticle seeds in the reaction mixture. A higher concentration of $\mathrm{Ag}$ nanoparticle seeds yielded smaller $\mathrm{Au}-\mathrm{Ag}$ nanodendrites with respect to their outer diameters and size of the branches on the nanodendrite surfaces. Besides, a series of $\mathrm{Cu}-\mathrm{Au}$ nanostructures (i.e. nanowires, nanotubes, and nanotriangles) were developed by our research group via a two-step approach. ${ }^{155}$ Firstly, the disproportionation reaction of the $\mathrm{Cu}^{+}$-oleylamine complex for the synthesis of $\mathrm{Cu}$ nanowires and the subsequent reaction with the $\mathrm{Au}^{3+}$ precursor at different temperatures to form the bimetallic $\mathrm{Cu}-\mathrm{Au}$ nanostructures were carried out. The controlled addition of the $\mathrm{Au}^{3+}$ precursor to the $\mathrm{Cu}$ 

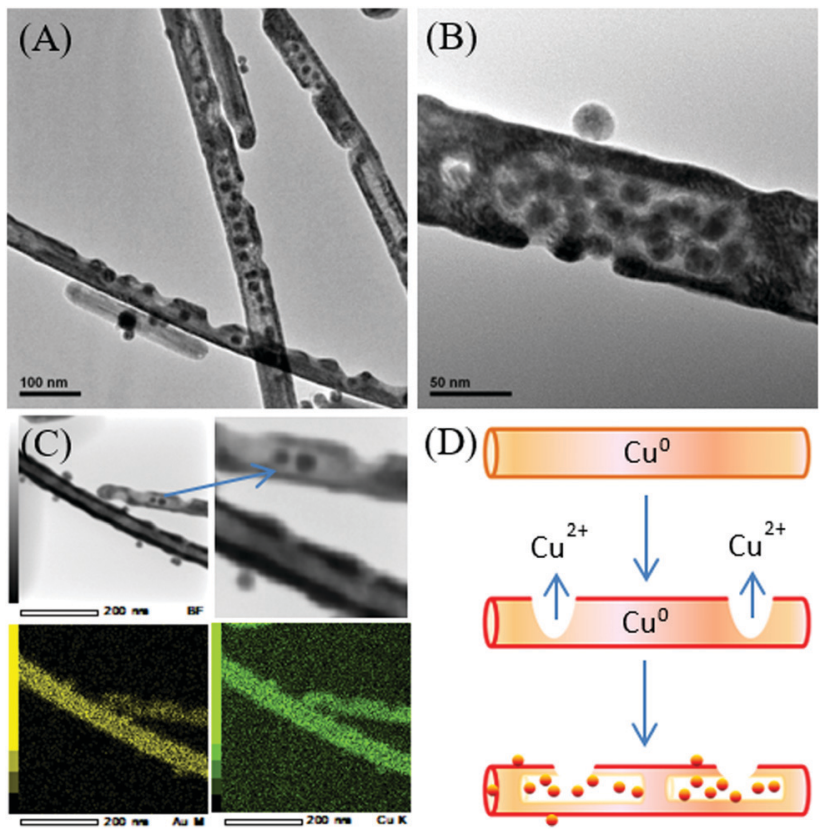

Fig. 9 (A and B) TEM images of bimetallic Cu-Au nanotubes. (C) TEM EDX mapping. (D) Schematic formation of $\mathrm{Cu}-\mathrm{Au}$ nanotubes with $\mathrm{Au}$ incorporated nanoparticles. Reproduced from ref. 155 with permission from the Royal Society of Chemistry.

nanowires dispersion resulted in galvanic replacement due to the electrochemical potential difference between $\mathrm{Cu}$ and $\mathrm{Au}$. In this process, the control of the reaction temperature was critical to yield the $\mathrm{Cu}-\mathrm{Au}$ nanostructures of a desired morphology. Notably, the appropriate reaction temperature of $140{ }^{\circ} \mathrm{C}$ yields $\mathrm{Cu}-\mathrm{Au}$ nanotubes through the surface passivation of the $\mathrm{Cu}$ nanowires when $\mathrm{Cu}^{0}$ is oxidized to a higher oxidation state of $\mathrm{Cu}^{2+}$ while $\mathrm{Au}^{3+}$ is reduced to $\mathrm{Au}^{0}$ (Fig. 9).

\section{Biomedical applications}

The development of metal and bimetallic functional nanostructures has progressed tremendously over the past decades due to their unique structural features and outstanding properties, particularly in the field of viable biomedical materials and research in nanotechnology. The following discussions focus on a diverse range of available coinage metal nanostructures as a promising platform for a wide variety of bio-applications such as antibacterial activity, SERS-based detection, and electrochemical sensing.

\subsection{Antibacterial activities}

Bacteria are among the most diverse groups of living organisms that differ in size, shape, habitat, and metabolism. These living organisms are capable of adapting to a great variety of ecological environments. On top of this, the serious problem is the bacterial adhesion to material surfaces and the subsequent formation of biofilms, which cause a major challenge in healthcare and industrial applications. As such, the prevention of bacterial attachment and biofilm growth is crucial, which has stimulated much efforts in the development of antibacterial metals. In general, antibacterial metals such as $\mathrm{Cu}, \mathrm{Ag}$, and $\mathrm{Au}$ are potentially used as a type of antibacterial agent. ${ }^{156-160}$ Significant progress in coinage metals relating to their antibacterial properties has been made through the design of metallic nanostructures as well as the understanding of their biological activity, structural, and functional changes induced in bacterial cells. ${ }^{158,160-165}$

3.1.1. Ag nanostructures in antibacterial activities. The size, shape, and the surface chemistry of the metallic nanostructures play an important role in the biocidal activity. For instance, the dimension of $\mathrm{Ag}$ nanoparticles influences the antibacterial activity and studies have shown that smaller particles impart a greater antibacterial activity. In the work of Sharma and co-workers, it was shown that $\mathrm{Ag}$ nanoparticles of $25 \mathrm{~nm}$, synthesized by the reduction by maltose, exhibited the highest antibacterial activity among all the other sizes up to $450 \mathrm{~nm} .{ }^{156}$ Briefly, Ag nanoparticles were prepared from the chemical reduction of $\left[\mathrm{Ag}\left(\mathrm{NH}_{3}\right)_{2}\right]^{+}$complex by four different saccharides (i.e., glucose, galactose, maltose, and lactose) to produce a wide range of particle sizes $(25-450 \mathrm{~nm})$ with narrow size distributions for antibacterial performance evaluation. It was pointed out that the bactericidal effect of $\mathrm{Ag}$ nanoparticles was not limited to the inhibition of bacterial growth but also to the killing of bacteria. Ivask et al. demonstrated that the $10 \mathrm{~nm}$ citrate-coated $\mathrm{Ag}$ nanoparticles significantly increased the toxicity toward Escherichia coli (E. coli) bacteria among the 20 and $40 \mathrm{~nm} \mathrm{Ag}$ nanoparticles. ${ }^{166}$ The size-dependent antibacterial activity of $\mathrm{Ag}$ nanoparticles was also demonstrated in the particle size of 5-100 $\mathrm{nm}$ against various bacterial strains, such as E. coli MTCC 443, E. coli MTCC 739, Bacillus subtilis (B. subtilis) MTCC 441, and Staphylococcus aureus (S. aureus) NCIM 5021. ${ }^{59}$ In particular, the antibacterial efficacy increased with smaller sized particles of less than $10 \mathrm{~nm}$, as revealed through delayed bacterial growth kinetics. It was anticipated that smaller particles have a larger surface area available for interaction, thus exhibiting greater bactericidal effect than the larger particles; therefore it is highly desirable to develop methods for the synthesis of monodisperse $\mathrm{Ag}$ nanoparticles with small size.

From these reports, $\mathrm{Ag}$ has been shown to display strong toxicity to an extensive range of microorganisms. Generally, the bactericidal activity is attributed to the Ag nanoparticles, which may attach and anchor to the surface of the bacterial cell wall and cause disruption of the cell function such as permeability and respiration, even cell death. ${ }^{167}$ Some studies have also shown that Ag nanoparticles are capable of penetrating inside the bacteria and causing further damage as well as the release of $\mathrm{Ag}$ ions from the nanoparticles, which promote the bactericidal activity. ${ }^{59,168}$ In addition to the size-dependent bactericidal properties of the nanoparticles, different shapes of $\mathrm{Ag}$ nanostructures may exhibit diverse antibacterial activities. A report has shown that truncated triangular $\mathrm{Ag}$ nanoplates exhibited a higher antibacterial activity against $E$. coli, relative to spherical, rod shaped nanoparticles, and also $\mathrm{Ag}$ ions. ${ }^{161}$ Similarly, $\mathrm{Ag}$ nanodendrites displayed stronger antimicrobial activities against E. coli, Candida albicans, and S. aureus, which were 
10 times higher than that of Ag nanoparticles. These studies suggest that the antimicrobial efficiency of $\mathrm{Ag}$ is size- and shape-dependent; in particular, $\mathrm{Ag}$ nanostructures with highly active surfaces potentially function as multiple antibiotics. ${ }^{169}$

3.1.2. Au and Au-Ag nanostructures in antibacterial activities. In comparison to $\mathrm{Ag}$, studies of the effect of $\mathrm{Au}$ nanostructures on the antibacterial activity are limited possibly due to the low toxicity of $\mathrm{Au}$ to the biological systems. In this context, Au nanoparticles exhibited different antibacterial effects against Gram-negative bacteria (i.e., E. coli) by changing the surface modification agents. Surface modification determines the surface charge and chemical properties of the nanoparticles, which is crucial for bacteria interaction. It has been demonstrated that cationicallyfunctionalized $\mathrm{Au}$ nanoparticles were more toxic than anionically-capped ones, ${ }^{157,170}$ for instance, polyallylamine hydrochloride-functionalized $\mathrm{Au}$ nanoparticles caused cell lysis while the citrate-capped Au nanoparticles did not. The antibacterial studies were also extended to bimetallic $\mathrm{Au}-\mathrm{Ag}$ nanostructures against a series of different bacteria. The $\mathrm{Au} @ A g$ core-shell nanoparticles prepared by seed-mediated reduction method were found to possess higher efficacy as compared to monometallic $\mathrm{Ag}$ nanoparticles for inhibiting Gram-negative bacterial growth. ${ }^{171}$ The enhanced antibacterial activity of Au@Ag core-shell nanoparticles could be attributed to the highly active surface $\mathrm{Ag}$ atoms in the shell around the $\mathrm{Au}$ core and/or electronic effect in bimetallic nanoparticles. Wang et al. studied the antibacterial effect of $\mathrm{Au}-\mathrm{Ag}$ nanostructures with hollow interiors and porous walls (i.e., nanocage) synthesized by the galvanic replacement of $\mathrm{Ag}$ nanocubes. In this work, the molar ratios of $\mathrm{Ag}$ to $\mathrm{Au}$ were tailored by varying the concentration of the $\mathrm{HAuCl}_{4}$ solution and the bimetallic nanostructures were then subjected to the antibacterial activity study. The bacterial cells (i.e., E. coli, E. faecalis, $S$. mutans, and $S$. aureus) that were treated with the $\mathrm{Au}-\mathrm{Ag}$ nanocages (molar ratio of $\mathrm{Ag} / \mathrm{Au}=2$ ) lost most of their original morphology and collapsed after incubation. The stronger antibacterial effect has been proposed for the electronic effects brought about by the addition of Au atoms into the Ag nanocubes. Because the $\mathrm{Au}$ atom has a higher electronegativity than the $\mathrm{Ag}$ atom, it can withdraw electrons from the $\mathrm{Ag}$ atom and probably make the free energy of the $\mathrm{Ag}$ atoms high and consequently more reactive compared to Ag nanocubes of a similar size. ${ }^{173}$ Through this observation, it is evident that the $\mathrm{Au}-\mathrm{Ag}$ nanocages exhibited broad-spectrum antibacterial properties potentially through the destruction of the cell membrane, production of reactive oxygen species, and induction of cell apoptosis (Fig. 10). ${ }^{172}$

3.1.3. Cu nanostructures in antibacterial activities. Considering the high cost associated with $\mathrm{Ag}$ and Au-related materials, $\mathrm{Cu}$ nanostructures offer a more economical solution. A report has shown that polymeric nanocomposites with $\mathrm{Cu}$ nanoparticles was capable for the controlled-release of the $\mathrm{Cu}$ species to inhibit the growth of living organisms, such as fungi and other pathogenic microorganisms. ${ }^{174}$ Tamayo et al. studied the antibacterial properties of $\mathrm{Ag}$ and $\mathrm{Cu}$ nanoparticles in polyethylene nanocomposites against Gram-positive pathogenic bacteria
(A)

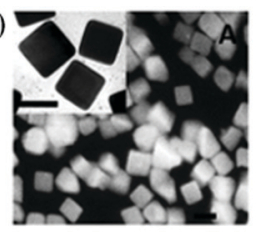

Ag NPs

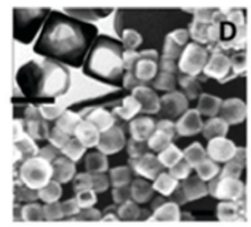

GSNC-3

(B)

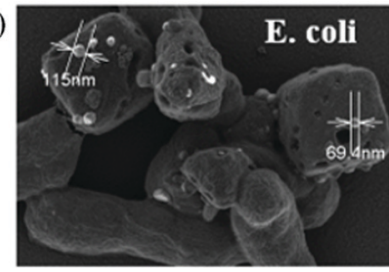

(C)

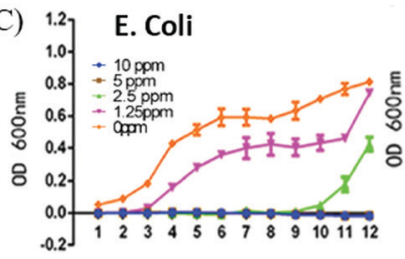

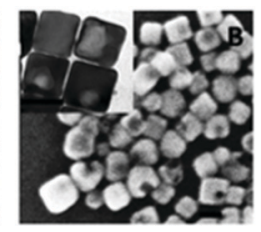

GSNC-10

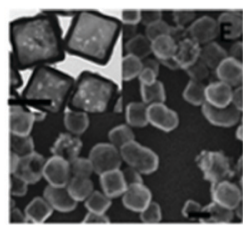

GSNC-2

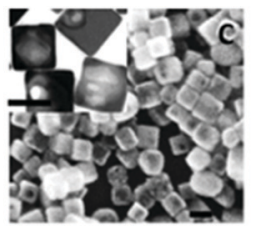

GSNC-7
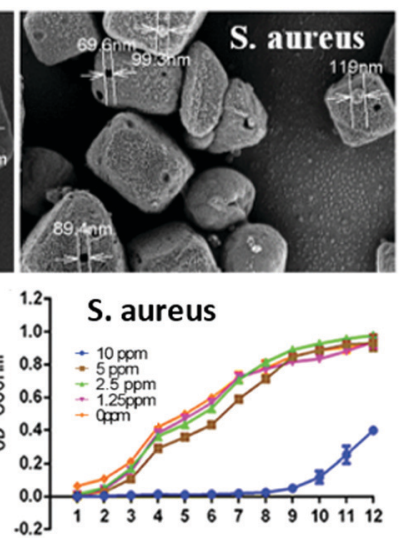

Fig. 10 (A) SEM and TEM (inset) images of Au-Ag nanocages (scale bar is $50 \mathrm{~nm}$ ) produced by reacting $\mathrm{Ag}$ nanocubes with varying amount of $\mathrm{HAuCl}_{4}$ to tailor different molar ratios of $\mathrm{Ag} / \mathrm{Au}$. (B) SEM images of $E$. coli and $S$. aureus to indicate cavities on the membrane, which matches with the $\mathrm{Au}-\mathrm{Ag}$ nanocages, and $(\mathrm{C})$ the corresponding $E$. coli and S. aureus growth curve with different concentrations of $\mathrm{Au}-\mathrm{Ag}$ nanocages. Reproduced from ref. 172 with permission from the Royal Society of Chemistry.

(i.e., Listeria monocytogenes). ${ }^{175}$ The increased antibacterial effect from $\mathrm{Cu}$ when compared with $\mathrm{Ag}$ nanoparticles was associated with $\mathrm{Cu}$ ion release, possibly due to the high susceptibility of $\mathrm{Cu}$ to oxidation. The ions released caused the membrane deformation of bacteria, promoted their entry into the bacteria, and changed the bacterial enzymatic functions. Likewise, $\mathrm{Cu}$ nanoparticles displayed the antibacterial properties against Gram-negative bacteria (i.e., E. coli). ${ }^{176-178}$ The $15 \mathrm{~nm} \mathrm{Cu}$ nanoparticles exhibited antibacterial properties by adhering to the bacterial cell wall, penetrating to the cell membrane, and imparting damage to the cell, eventually leading to cell death. ${ }^{158}$ The bactericidal activity is concentration-dependent, which is related to the release of $\mathrm{Cu}$ ion concentration. Besides, the shape of $\mathrm{Cu}$ nanostructures also plays a vital role in the bactericidal activity. In this study, a series of $\mathrm{Cu}$ nanostructures of different shapes and sizes (i.e., nanorod, nanotriangular, spherical, and fibrous-shape) were synthesized by the chemical reduction method by varying the precursor parameters such as the type of $\mathrm{Cu}$ salts and reducing agents. The finding revealed that the fibrous-shape $\mathrm{Cu}$ nanostructures prepared by reducing with $\mathrm{NaOH}$, in general, possessed stronger antimicrobial properties against both Gram-positive and Gram-negative food-borne pathogens than those synthesized with ascorbic acid. ${ }^{179}$ 


\subsection{SERS sensing}

SERS is a surface-sensitive technique that offers several orders of magnitude increase in the Raman intensity for detecting and identifying chemical and biological compounds at low concentrations. Since the discovery of the SERS effect in 1974 by Fleischman and colleagues, ${ }^{180}$ great efforts have been made toward understanding of the enhancement process. It is widely accepted that SERS enhancement is attributed to two primary mechanisms-electromagnetic field enhancement and chemical enhancement. The electromagnetic mechanism provides the main role for the SERS effect, which primarily results from the amplification of the local electromagnetic field due to localized surface plasmon resonance while the chemical enhancement is related to charge transfer between the chemisorbed molecule and the Raman-active platform. ${ }^{181}$ The signal enhancement enables detection with extraordinary sensitivity even at the single-molecule level. ${ }^{182}$

3.2.1. Monometallic Ag and Au nanostructures in SERS sensing. Noble metals $\mathrm{Ag}$ and $\mathrm{Au}$ have been widely investigated as the highly active SERS substrates. ${ }^{183,184}$ In particular, Ag nanoparticles afford strong SERS signals from the ultraviolet to the near infrared region, and $\mathrm{Au}$ nanoparticles show good biocompatibility, which render them more suitable for detection in biomedical fields. ${ }^{185}$ The particle size and shape are important factors contributing to the plasmonic properties, while the increased sharpness and roughness in the metal nanostructures amplify the SERS signal because of the presence of plasmonic hot spots, which increases the surrounding electrical field. ${ }^{181}$

To date, various nanostructures with different morphologies including nanorods, ${ }^{186-188}$ nanotriangles, ${ }^{189,190}$ nanocubes, ${ }^{191-193}$ nanostars, ${ }^{112,194}$ nanocages, ${ }^{195,196}$ and some other branched morphologies ${ }^{197-200}$ have been suggested for the fabrication of potential SERS-active platforms. For example, the correlation of the shape to SPR and SERS was studied with $\mathrm{Au}$ nanorods of different aspect ratios, which were prepared from the seedmediated approach. ${ }^{201}$ The Au nanorods are particularly interesting because their anisotropy in dimensions lead their SPR to split into a transverse mode and a longitudinal mode. The longitudinal plasmonic bands can be finely tuned across the visible to the near-infrared region by increasing the aspect ratio of the nanorods. This spectral range $(650-900 \mathrm{~nm})$ is useful for developing nearinfrared SERS nanoprobes in bioanalytical applications such as biomedical imaging and sensing. The shape-dependent SERS of the Au nanostructures has also been investigated on Au nanostars and spherical nanoparticles. ${ }^{202}$ This study illustrated that the spherical nanoparticles displayed a surface plasmon band centered at $527 \mathrm{~nm}$, while star-shaped colloids of similar size exhibited a strongly red-shifted surface plasmon resonance with the maximum absorbance at $730 \mathrm{~nm}$ in the near-infrared region. The red-shifted band was associated to the plasmon mode localized at the nanostar tips. The SERS enhancement by the nanostars was enhanced several-fold when tested on analytes, thus demonstrating the high electromagnetic fields achieved by using nanostars as the optical enhancers.

A series of Au nanocrystals have been prepared by the seedmediated approach and the facet-controlled products with four different shapes were assembled into an ordered array for the examination of facet-dependent SERS performance. ${ }^{203}$ The products obtained were cubic, concave cubic, octahedral, and rhombic dodecahedral Au nanocrystals, encased by (100), (200), (111), and (110), respectively. Among the Au nanocrystals, rhombic dodecahedra plasmonic monolayers exhibited the strongest SERS activity, with an analytical enhancement factor up to $1.2 \times 10^{6}$. In another SERS application of $\mathrm{Au}$ nanostructures, $\mathrm{Au}$ nanocages have been applied to serve as carriers for targeted and triggered drug delivery, while SERS was employed to monitor the release of Raman-active molecules such as dyes and drugs from the plasmonic nanocages. ${ }^{195}$ In this study, the Au nanocages obtained from the galvanic replacement of $\mathrm{Ag}$ nanocubes were loaded with the phase change material (1-tetradecanol) and Nile red or chemotherapeutic drug molecules (e.g., doxorubicin), followed by the release of encapsulated drugs along with melted 1-tetradecanol by thermal means. The release of the cargo from the Au nanocages was monitored by SERS, of which the intensity of the SERS spectra displayed a monotonic decrease in the intensity with longer exposure to elevated temperature, indicating the progressive release of the cargo from the $\mathrm{Au}$ nanocages. On the contrary, the control sample showed no change in the SERS intensity at room temperature (Fig. 11). In the SERS-based sensing applications, significant investigation have been conducted towards the detection of biomolecules and proteins. $^{204-208}$ The SERS substrate composed of concave Au nanocubes was supported on a polydimethylsiloxane film and fabricated as a SERS active platform for protein detection (i.e., insulin and cytochrome $c$ ). ${ }^{209}$ The close assembly of the concave nanocubes promoted the formation of nanoholes through faceface and face-corner interactions between adjacent particles, which played the role of traps for effective protein detection. Once the proteins were trapped into the concave nanocubes arrays, the

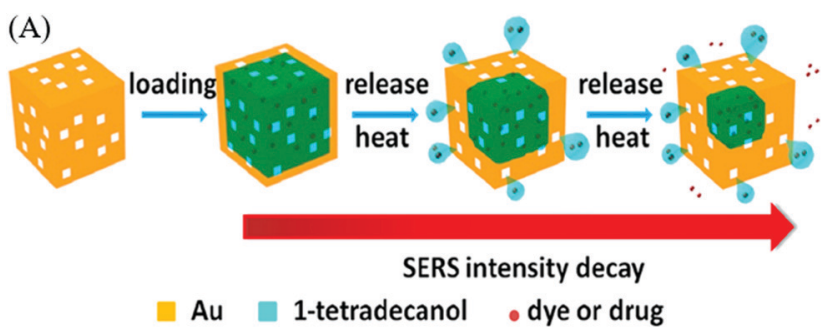

(B)

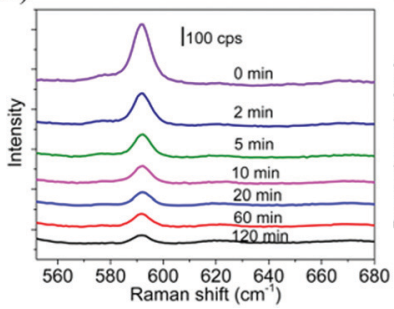

(C)

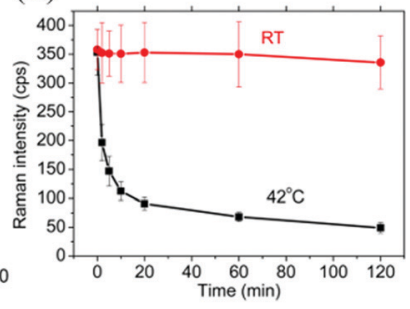

Fig. 11 (A) Scheme illustrating drug delivery using Au nanocage. (B) Raman spectra of $\mathrm{Au}$ nanocages at different time intervals. (C) Raman intensity from Au nanocages exposed to water at $42{ }^{\circ} \mathrm{C}$ and water at room temperature during different time intervals. Reproduced from ref. 195 with permission from the American Chemical Society. 
molecule experienced an electric field enhancement, which was expected to amplify the vibrational modes of a large part of the protein.

3.2.2. Bimetallic Au-Ag nanostructures in SERS sensing. Apart from the synthesis of monometallic nanostructures for SERS applications, bimetallic nanostructures have been extensively explored as promising SERS substrates. For example, the unique bimatallic Au@AuAg yolk-shell nanostructures with tunable interior gap, which were fabricated through seedmediated and galvanic replacement, have been shown to exhibit great SERS capability. ${ }^{210}$ The interior gap that induced strong electromagnetic coupling in the Au@AuAg yolk-shell nanostructures could generate intensive electromagnetic fields in comparison to individual $\mathrm{Au}$ triangular nanoplates or solid Au@Ag core-shell triangular nanoplates, producing more hot spots in the interior gap. Also, the intensive plasmonic coupling effect that occurred between the tips of the core and the concave of the inner wall of the shell played an important role in the observed SERS enhancement. The careful design of SERS substrates enables the detection of bioanalytes at very low concentration. In this context, SERS-based immunoassay for the detection of cardiorenal syndrome biomarkers was demonstrated using $\mathrm{Au}-\mathrm{Ag}$ bimetallic nanostars, which were synthesized via galvanic replacement. ${ }^{211}$ The Raman reporter molecule-labeled $\mathrm{Au}-\mathrm{Ag}$ nanostars were used as the nanotags and a $3-\mathrm{D}$ ordered macroporous $\mathrm{Au}-\mathrm{Ag}-\mathrm{Au}$ plasmonic array was employed as the substrate. The multibranched structure and bimetallic composition displayed high SERS stability and enhancement, which allowed SERS-based protein detection with a detection limit of the fg $\mathrm{mL}^{-1}$ level. Likewise, The SERS-based immunoassay for the detection of proteins was constructed using $\mathrm{Au}-\mathrm{Ag}$ bimetallic nanoparticles with label molecules as the nanotags and the $\mathrm{Au}-\mathrm{Ag}$ bimetallic film as the substrate. ${ }^{212}$ The bimetallic nanoparticles with enriched Ag content increased the SERS enhancement, which allowed rabbit IgG detection with a detection limit of

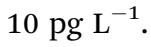

3.2.3. $\mathrm{Cu}$ and $\mathrm{Cu}-\mathrm{Au}$ nanostructures in SERS sensing. Among coinage metals, $\mathrm{Cu}$ has the advantage of reducing the cost and abundance but the relatively weak enhancement factor of $\mathrm{Cu}$ limits its application as a SERS platform. To improve the SERS performance of $\mathrm{Cu}$ nanomaterials, hierarchical 3-D structured materials have been constructed via the templatebased approach, providing electromagnetic field enhancement through the concentrated hot spots at the sharp tips and gaps. $^{213}$ The as-designed 3-D Cu nanostructures exhibited superior SERS performance with an high enhancement factor up to $10^{7}$, indicating a promising SERS substrate. The application of $\mathrm{Cu}$ nanostructures for Cu-SERS substrates has been used as a detection tool in chemical analysis. ${ }^{214}$ For example, the Cu-SERS substrates were employed in the detection of various cephalosporin antibiotics (i.e., ceftriaxone, cefazolin, and cefoperazone) in urine, with a high Raman enhancement efficiency, which is comparable to that of $\mathrm{Ag}$ nanoparticles and significantly higher than that of $\mathrm{Au}$ nanoparticles. ${ }^{215}$ The Cu-SERS substrates are composed of $\mathrm{Cu}$ nanoparticles obtained via the reduction of $\mathrm{Cu}^{2+}$ ions by hydrazine hydrate in the presence of citrate ions. Despite $\mathrm{Cu}$ nanoparticles exhibiting poorer temporal stability relative to $\mathrm{Ag}$ and $\mathrm{Au}$ nanoparticles, the application of freshly prepared $\mathrm{Cu}$-SERS substrates resulted in satisfactory accuracy (recovery = $93-107 \%)$. The main limitation of the $\mathrm{Cu}$ material is the susceptibility to oxidation upon exposure to air. Apart from using a temporal protector $\left(\right.$ e.g., $\left.\mathrm{CaCO}_{3}\right)$ to minimize the interaction between $\mathrm{Cu}$ nanoparticles and air oxygen, ${ }^{216}$ one effective strategy is to fabricate $\mathrm{Cu}$-based binary alloy nanostructures to improve the stability in air and the SERS performance. For example, the $\mathrm{Cu}-\mathrm{Au}$ alloy was employed as the SERS substrate using the template-based approach to produce $\mathrm{Cu}-\mathrm{Au}$ nanotubes with a five-fold twinned structure. ${ }^{217}$ The SERS measurement has shown that the enhancement of SERS on the $\mathrm{Cu}-\mathrm{Au}$ alloy nanotubes was at least three times higher than that on the $\mathrm{Cu}$ nanowires using 4-mercaptopyridine solution as the probe molecule. It was pointed out that a large number of hot spots were produced at the intersection of the nanotubes for contributing to the SERS enhancement.

\subsection{Electrochemical sensing}

Electrochemical sensing is commonly used for metabolic disorder detection in human body. ${ }^{218}$ A metabolic disorder occurs when abnormal chemical reactions in the body interfere with the normal metabolic process and cause either accumulation of large amounts of one metabolite or a deficiency of one or more metabolites in the body to stay healthy. For example, high level of uric acid, oxalic acid, and citric acid in the urine is associated with an increased risk of forming kidney stones, while an excess level of creatinine in the bloodstream and urine can be an indicator of impaired kidney function or kidney disease. Elevated levels of $\mathrm{H}_{2} \mathrm{O}_{2}$ are generated from many fundamental biological oxidative reactions as a by-product or an intermediate product, signifying the existence of an illness. Among these metabolic disorders, diabetes is the most common metabolic disease that is caused by the high level of blood glucose in the body over a prolonged period. Therefore, the study on glucose sensing is of practical significance and the development of an ideal glucose sensor is the most important issue in the biosensor field.

3.3.1. Non-enzymatic glucose sensing. Over the past five decades, the development of enzymatic glucose sensors has prevalently dominated the glucose sensing industry due to the superior selectivity and negligible toxicity of enzyme-based electrodes. However, enzymatic glucose sensors are also often associated with complicated enzyme immobilization procedures, critical operating conditions such as optimum temperature, humidity, and $\mathrm{pH}$ that hinder their further development. ${ }^{219,220}$ Great efforts have been made for constructing non-enzymatic electrochemical glucose biosensors using metal-based nanostructures to alleviate the limitations of enzymatic glucose sensors. $^{221}$

The catalytic effects of metal-based nanostructured materials are highly dependent on the generation of electrocatalytically active sites and the enlargement of the surface area, which are 
closely related to the composition, structure, and morphology of the metal nanomaterials. For example, the electrochemical glucose sensor comprised of self-assembled $\mathrm{Au}$ nanoparticles on a 3-D silicate network demonstrated efficient glucose oxidation at a low potential $(0.16 \mathrm{~V} v s . \mathrm{Ag} / \mathrm{AgCl})$ in phosphate buffer solution at $\mathrm{pH} 9.2$, showing a detection limit of $50 \mathrm{nM}$. There are also other Au nanostructures such as Au nanotube arrays ${ }^{222}$ and $\mathrm{Au}$ nanowire arrays, ${ }^{223}$ which have been studied as electrochemical glucose sensors due to their unique nanostructures. The high surface area of $\mathrm{Au}$ electrodes facilitated the large electrochemical response of glucose, which enabled glucose detection sensitively and selectively in the presence of electrochemical interference species. Despite the advantages of sensitivity toward glucose oxidation, the high-cost noble metal makes them less competitive in the practical application for the growing demand.

In search of new materials, $\mathrm{Cu}$ and their bimetallic nanostructures such as $\mathrm{Cu}-\mathrm{Au}$ are promising materials for the mass production of glucose sensors in terms of the cost effectiveness, good sensitivity, and interference-free nature. To date, a wide range of $\mathrm{Cu}$ nanostructures such as $\mathrm{Cu}$ nanoparticles, $\mathrm{Cu}$ nanowires, $\mathrm{Cu}$ nanobelts, and $\mathrm{Cu}$ polyhedron pattern have been synthesized and studied as glucose sensors. ${ }^{220,224}$ Typically, during the oxidation of glucose to glucolactone on the $\mathrm{Cu}$ electrode, it is proposed that the catalytic component is $\mathrm{Cu}$ (III) oxyhydroxide species, the oxidized partner to the $\mathrm{Cu}(\mathrm{OH})_{2} /$ $\mathrm{CuOOH}$ redox couple; hence, the catalytic reaction is highly dependent on the $\mathrm{Cu}(\mathrm{II}) / \mathrm{Cu}$ (III) redox couple. Our research group has developed a non-enzymatic glucose sensor based on various $\mathrm{Cu}-\mathrm{Au}$ nanostructures from $\mathrm{Cu}$ nanowires template (Fig. 12). The bimetallic $\mathrm{Cu}-\mathrm{Au}$ nanostructures exhibited superior sensing performance compared to the monometallic $\mathrm{Cu}$ nanowires, which clearly demonstrated their synergistic effect for electrocatalytic glucose oxidation. In particular, the sensing capability was certainly evident from the significantly improved electrocatalytic activity after adding a small quantity of $\mathrm{Au}$ of about $3 \mathrm{mmol} \%$ to the $\mathrm{Cu}$ nanowires. Furthermore, the high aspect ratio $\mathrm{Cu}-\mathrm{Au}$ nanotubes with a large surface area provided abundant electroactive sites and facilitated mass transport, thereby improving the sensor response among other $\mathrm{Cu}-\mathrm{Au}$ nanostructures.

3.3.2. Non-enzymatic $\mathrm{H}_{2} \mathrm{O}_{2}$ sensing. The work of coinage metal nanostructures in non-enzymatic electrochemical sensing has also been demonstrated for $\mathrm{H}_{2} \mathrm{O}_{2}$ detection. An amperometric $\mathrm{H}_{2} \mathrm{O}_{2}$ sensor was reported based on highly dense $\mathrm{Ag}$ nanowires that were synthesized by a polyol process and their assembly with a chitosan film. In this way, the sensor offered several benefits including high electrical conductivity and good electrocatalytic activity, which were attributed to the inherent properties of the $\mathrm{Ag}$ nanowires as well as the architectural stability through the chitosan polymer film. ${ }^{225}$ In another example, the fabrication of the non-enzymatic electrochemical $\mathrm{H}_{2} \mathrm{O}_{2}$ sensor was developed based on hierarchical Ag microflowers via the precipitation method. ${ }^{226}$ A series of the $\mathrm{Ag}$ microstructures ranging from ball-like to flower-like were synthesized and their morphologies were tuned by different dicarboxylic acids such as

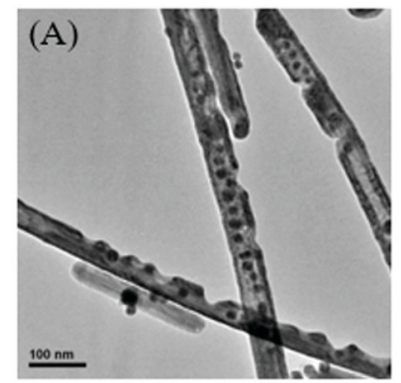

(C)

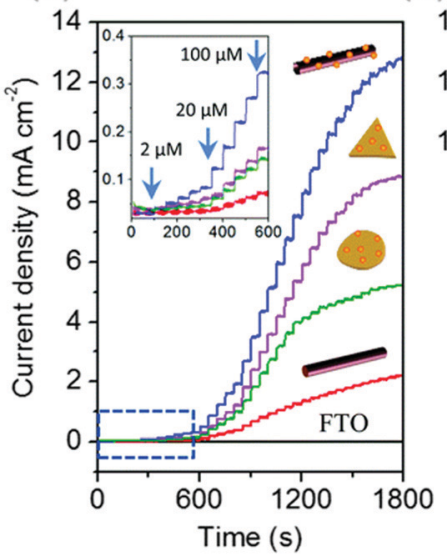

(B)

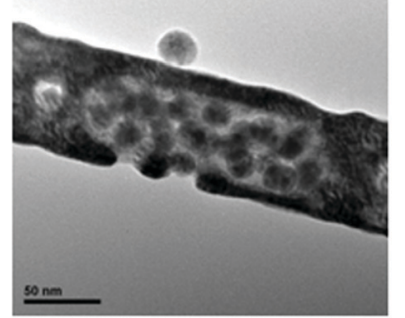

(D)

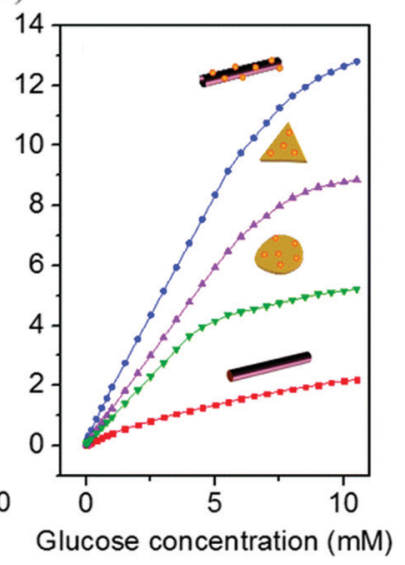

Fig. 12 ( $A$ and B) TEM images of bimetallic $\mathrm{Cu}-\mathrm{Au}$ nanotubes. ( $C$ and $D$ ) Electrocatalytic activities of $\mathrm{Cu}$ nanowires and $\mathrm{Cu}-\mathrm{Au}$ nanostructures electrodes for glucose oxidation. Reproduced from ref. 155 with permission from the Royal Society of Chemistry.

structure-directing agents. Among the Ag microstructures, the rose flower-like microstructures exhibited the highest electrocatalytic activity for $\mathrm{H}_{2} \mathrm{O}_{2}$ sensing, showing 24 times higher sensitivity $\left(2.4 \mathrm{mM}^{-1} \mathrm{~cm}^{-2}\right.$ ) as compared to ball-shaped structures. The great sensitivity was attributed to the unique microstructures, offering outstanding active surface areas and a faster detachment rate of the in situ generated oxygen bubbles from the surface. Besides, the monitoring of $\mathrm{H}_{2} \mathrm{O}_{2}$ release in live cells was studied based on the autocatalytic oxidation reaction of $\mathrm{Ag}$ nanoparticles. ${ }^{227}$ The work demonstrated the decomposition of $\mathrm{H}_{2} \mathrm{O}_{2}$ in the presence of $\mathrm{Ag}$ nanoparticles, which released $\mathrm{Ag}^{+}$ and dissolved $\mathrm{Ag}$ nanoparticles progressively. As a result, the $\mathrm{H}_{2} \mathrm{O}_{2}$-induced $\mathrm{Ag}$ nanoparticle dissolution was monitored and demonstrated to be a good indicator of $\mathrm{H}_{2} \mathrm{O}_{2}$ concentration. In another example of $\mathrm{H}_{2} \mathrm{O}_{2}$ detection, bimetallic $\mathrm{Cu}$-Au nanowires have been fabricated and studied as $\mathrm{H}_{2} \mathrm{O}_{2}$ biosensors for detecting $\mathrm{H}_{2} \mathrm{O}_{2}$ secretion in live cells. ${ }^{228}$ The $\mathrm{Cu}-\mathrm{Au}$ nanowires were synthesized by the wet chemical route while compositions of the bimetallic nanowire were tuned by the dissolution of the $\mathrm{Cu}$ atoms from the $\mathrm{Cu}-\mathrm{Au}$ nanoalloy with the acid solution. The dealloying process increased the pore volume and offered abundant active sites for detecting the analyte, thus achieving a low detection limit of $\sim 2 \mathrm{nM}$ for $\mathrm{H}_{2} \mathrm{O}_{2}$. The superior sensing capability was ascribed to the synergetic effect resulting from the strong interaction among the $\mathrm{Cu}$ and $\mathrm{Au}$ electron cloud within the nanoalloy, which contributed to the fast renewal of the clean $\mathrm{Au}$ spots for $\mathrm{H}_{2} \mathrm{O}_{2}$ oxidation. 
Table 1 Coinage metal nanostructures synthesized using different methods

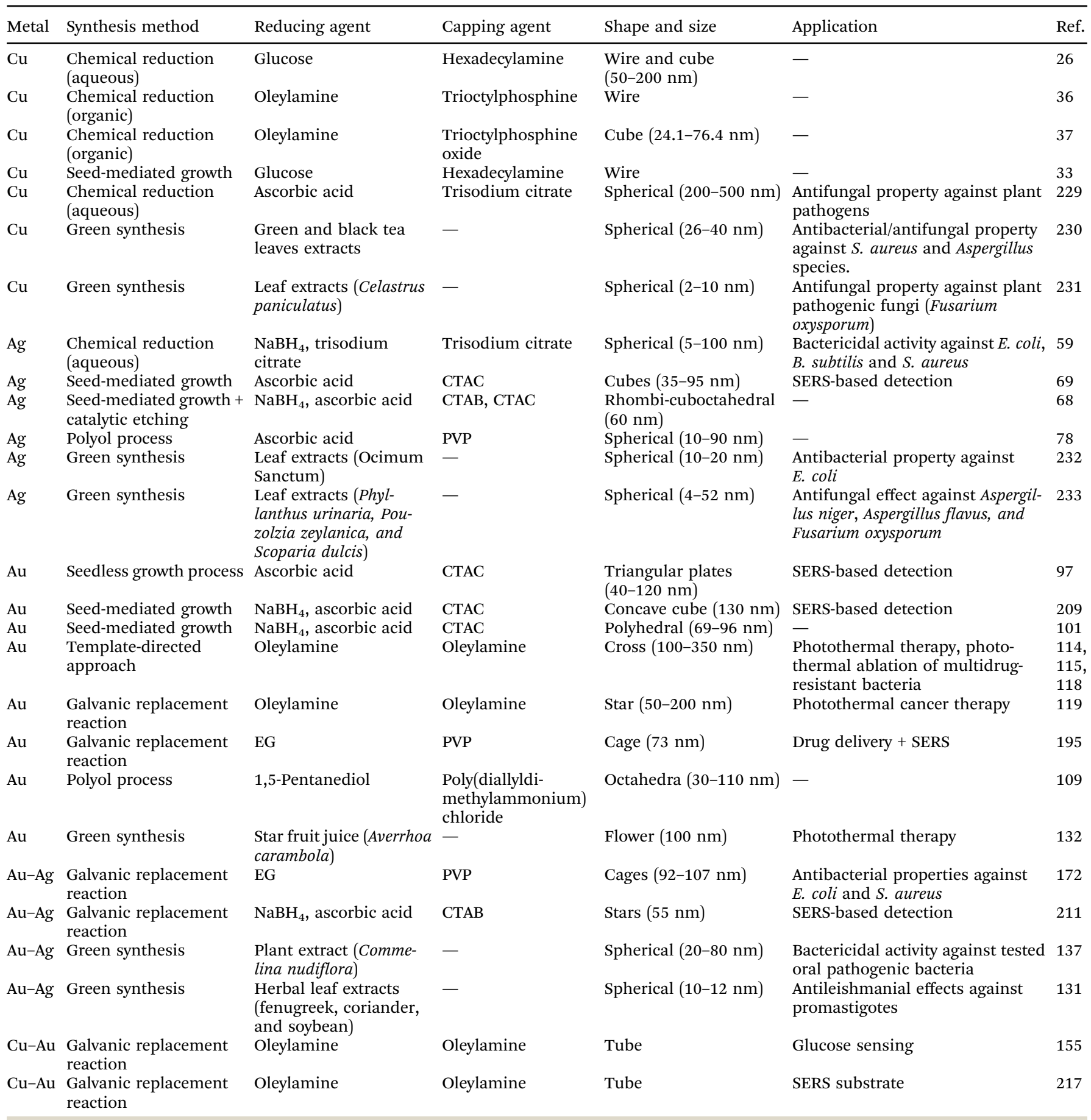

\section{Conclusions and future prospects}

The emergence of nanoscience and nanotechnology research have brought a large number of synthetic methods for the development of coinage metal nanostructures. As discussed in the review, these synthetic strategies such as the choice of a reducing agent, capping agent, the use of ionic impurity, secondary shape control agent (i.e., halide ions), and seedmediated approach provide a means to control the size, shape, composition, and consequently the properties of the metal to be tuned for the application. The functional properties of coinage metal nanostructures are correlated to their surface area, whose reducing agents and capping agents are important to control the size and prevent particle aggregation. Examples of reducing agents include (but are not limited to) sodium borohydride, sodium citrate, ascorbic acid, saccharides, and polyols, which play a major role in the conversion of metal salts into metal nanoparticles. Nevertheless, $\mathrm{Cu}$ nanostructures are 
often synthesized using a variety of organic-phase-based methods due to the highly oxidizing nature of the metal. On the other hand, citrate anions have been regarded as one of the most widely accepted reductants for the size-controlled synthesis of Ag nanoparticles owing to the slow crystal growth as a result of the interaction between $\mathrm{Ag}$ and citrate ions. Capping agents such as PVP have shown promising results in preventing particle aggregation, which provides good stability and size/shape uniformity through good adsorption of the carbonyl group and nitrogen atom of the pyrrolidone ring with the metal surface. In addition, the presence of the halide ions affects the overall crystal growth, resulting in the formation of different shapes of Au nanoparticles through selective binding on the crystal facets. As for bimetallic nanostructures, the galvanic replacement reaction represents a simple and versatile approach for engineering the architectural nanostructures with hollow interiors and porous walls.

The incorporation of coinage metal nanostructures in bioapplications has been extensively studied by the scientific community, of which the highlighted one in this review are the antibacterial activities, SERS-based detection, and electrochemical sensing. For instance, the size and shape of the metallic nanostructures have been finely tuned for the biocidal activity; in particular, the smaller particles with highly active surfaces potentially lead to higher antimicrobial efficiency. As for SERS sensing, the amplification of the SERS signal depends on a combination of the particle size, shape, as well as the increased sharpness and roughness in the metal nanostructures. In the context of electrochemical sensing, superior sensing performance has been demonstrated from the bimetallic nanostructures compared to the monometallic, which clearly exhibited the synergistic effect for electrocatalytic activity. Moreover, the porous nanostructures obtained from the dealloying process of the galvanic replacement reaction offer a larger surface area with abundant electroactive sites for detecting the analyte, thereby improving the sensor response. The properties of these coinage metal nanostructures are discussed here only to a limited extent and there are many promising applications in the chemical, energy, electronics, medicine, and healthcare fields. With regard to healthcare, the threat of the lethal Covid-19 viral infection has stressed healthcare systems around the world; coinage metal nanostructures may contribute significantly in the containment, diagnosis, and treatment of COVID-19. For example, Cu-based nanomaterials are potential candidates to be deployed for antiviral and antimicrobial nanocoatings, while $\mathrm{Au}$ and $\mathrm{Ag}$ nanomaterials are promising in drug delivery and therapeutics; in particular, $\mathrm{Ag}$ nanoparticles have significant virus-inhibitory properties.

To date, a large variety of protocols and approaches are available for the synthesis, assembly, fabrication, and the potential applications of coinage metal nanostructures. In the future, the challenge is to use such techniques reliably for largescale production. Nevertheless, more effort is needed toward the scalable routes of synthesis before promising demonstrations can be transitioned to commercialization. This includes the precise control of the coinage metal nanostructures properties (i.e., optical, mechanical, electrical, and chemical) for each technological application, which are determined by a set of physical parameters such as the size, shape, surface functionality, and composition. Moreover, these metal nanostructures may have a tendency to aggregate during the growth or post-processing, which may affect the specific functionality of the nanostructures. Among the nanostructures of coinage metals, the low producibility and long-term stability of the $\mathrm{Cu}$ nanostructures remains a grand challenge for their large-scale production and a comprehensive solution is still lacking even though some shortcomings have been addressed.

Despite the promising future of coinage metal nanostructures, the uncontrolled and unregulated use of these nanostructures in a diverse range of applications will likely lead to adverse impacts on the human health and environment. To enable the safer use of these metal nanostructures, the toxicological investigation of nanoparticles and surface coating as well as the in-depth understanding of their interactions with various biological compounds, cells, tissues, or bioenvironment are essential. Notably, these studies will provide important insight for the further investigation of the nanostructures in in vivo applications such as medical diagnostics and therapeutics. Moving forward, there is still room for the intensive investigation of new metallic/bimetallic systems because of the importance to develop multifunctional nanoparticles with numerous properties that enable greater ability than conventional nanoparticles in order to accomplish several objectives synergistically (Table 1).

\section{Conflicts of interest}

There are no conflicts to declare.

\section{Acknowledgements}

This work was supported by the Institute of Materials Research and Engineering (IMRE), Agency for Science, Technology and Research (A*STAR).

\section{References}

1 Z. Y. Zhou, N. Tian, J. T. Li, I. Broadwell and S. G. Sun, Chem. Soc. Rev., 2011, 40, 4167-4185.

2 K. Zhou and Y. Li, Angew. Chem., Int. Ed., 2012, 51, 602-613.

3 P. K. Jain, X. Huang, I. H. El-Sayed and M. A. El-Sayed, Acc. Chem. Res., 2008, 41, 1578-1586.

4 H. M. Chen and R.-S. Liu, J. Phys. Chem. C, 2011, 115, 3513-3527.

5 E. C. Dreaden, A. M. Alkilany, X. Huang, C. J. Murphy and M. A. El-Sayed, Chem. Soc. Rev., 2012, 41, 2740-2779.

6 S. E. Lohse and C. J. Murphy, J. Am. Chem. Soc., 2012, 134, 15607-15620.

7 C. N. Rao, H. S. Ramakrishna Matte, R. Voggu and A. Govindaraj, Dalton Trans., 2012, 41, 5089-5120. 
8 I. Khan, K. Saeed and I. Khan, Arabian J. Chem., 2019, 12, 908-931.

9 M. Faraday, Philos. Trans. R. Soc. London, 1857, 147, 145-181.

10 B. V. Enüstün and J. Turkevich, J. Am. Chem. Soc., 1963, 85, 3317-3328.

11 L. Au, Y. Chen, F. Zhou, P. H. Camargo, B. Lim, Z. Y. Li, D. S. Ginger and Y. Xia, Nano Res., 2008, 1, 441-449.

12 C. L. Bracey, P. R. Ellis and G. J. Hutchings, Chem. Soc. Rev., 2009, 38, 2231-2243.

13 J. Yang, X. Chen, X. Yang and J. Y. Ying, Energy Environ. Sci., 2012, 5, 8976.

14 G. Guisbiers, S. Mejia-Rosales, S. Khanal, F. Ruiz-Zepeda, R. L. Whetten and M. Jose-Yacaman, Nano Lett., 2014, 14, 6718-6726.

15 G. Darabdhara, B. Sharma, M. R. Das, R. Boukherroub and S. Szunerits, Sens. Actuators, B, 2017, 238, 842-851.

16 R. H. Morriss and L. F. Collins, J. Chem. Phys., 1964, 41, 3357-3363.

17 Y. Xia, Y. Xiong, B. Lim and S. E. Skrabalak, Angew. Chem., Int. Ed., 2009, 48, 60-103.

18 J. A. Dahl, B. L. Maddux and J. E. Hutchison, Chem. Rev., 2007, 107, 2228-2269.

19 Y. Xia, K. D. Gilroy, H. C. Peng and X. Xia, Angew. Chem., Int. Ed., 2017, 56, 60-95.

20 S.-Y. Zhang, M. D. Regulacio, K. W. Shah, T. Sreethawong, Y. Zheng and M.-Y. Han, J. Mol. Eng. Mater., 2014, 02, 1430001.

21 A. R. Tao, S. Habas and P. Yang, Small, 2008, 4, 310-325.

22 Y. Xia, Y. Xiong, B. Lim and S. E. Skrabalak, Angew. Chem., Int. Ed., 2009, 48, 60-103.

23 K. Zhou and Y. Li, Angew. Chem., Int. Ed., 2012, 51, 602-613.

24 P. D. Cozzoli, T. Pellegrino and L. Manna, Chem. Soc. Rev., 2006, 35, 1195-1208.

25 R. Costi, A. E. Saunders and U. Banin, Angew. Chem., Int. Ed., 2010, 49, 4878-4897.

26 M. Jin, G. He, H. Zhang, J. Zeng, Z. Xie and Y. Xia, Angew. Chem., Int. Ed., 2011, 50, 10560-10564.

27 D. Zhang, R. Wang, M. Wen, D. Weng, X. Cui, J. Sun, H. Li and Y. Lu, J. Am. Chem. Soc., 2012, 134, 14283-14286.

28 I. Lisiecki, F. Billoudet and M. P. Pileni, J. Phys. Chem., 1996, 100, 4160-4166.

29 B. K. Park, S. Jeong, D. Kim, J. Moon, S. Lim and J. S. Kim, J. Colloid Interface Sci., 2007, 311, 417-424.

30 Y. Chang, M. L. Lye and H. C. Zeng, Langmuir, 2005, 21, 3746-3748.

31 A. R. Rathmell, S. M. Bergin, Y. L. Hua, Z. Y. Li and B. J. Wiley, Adv. Mater., 2010, 22, 3558-3563.

32 S. Bhanushali, P. Ghosh, A. Ganesh and W. Cheng, Small, 2015, 11, 1232-1252.

33 Q. Q. Fu, Y. D. Li, H. H. Li, L. Xu, Z. H. Wang and S. H. Yu, Langmuir, 2019, 35, 4364-4369.

34 D. Mott, J. Galkowski, L. Wang, J. Luo and C. J. Zhong, Langmuir, 2007, 23, 5740-5745.

35 F. Cui, Y. Yu, L. Dou, J. Sun, Q. Yang, C. Schildknecht, K. Schierle-Arndt and P. Yang, Nano Lett., 2015, 15, 7610-7615.
36 E. Ye, S. Y. Zhang, S. Liu and M. Y. Han, Chem. - Eur. J., 2011, 17, 3074-3077.

37 H. Guo, Y. Chen, M. B. Cortie, X. Liu, Q. Xie, X. Wang and D.-L. Peng, J. Phys. Chem. C, 2014, 118, 9801-9808.

38 X. Huang, Y. Chen, C. Y. Chiu, H. Zhang, Y. Xu, X. Duan and Y. Huang, Nanoscale, 2013, 5, 6284-6290.

39 N. George, J. Faoagali and M. Muller, Burns, 1997, 23, 493-495.

40 B. S. Atiyeh, M. Costagliola, S. N. Hayek and S. A. Dibo, Burns, 2007, 33, 139-148.

41 C. Chen, L. Wang, H. Yu, J. Wang, J. Zhou, Q. Tan and L. Deng, Nanotechnology, 2007, 18, 115612.

42 J. Zhang, M. R. Langille and C. A. Mirkin, J. Am. Chem. Soc., 2010, 132, 12502-12510.

43 K. M. M. Abou El-Nour, A. a. Eftaiha, A. Al-Warthan and R. A. A. Ammar, Arabian J. Chem., 2010, 3, 135-140.

44 Z. Huang, X. Jiang, D. Guo and N. Gu, J. Nanosci. Nanotechnol., 2011, 11, 9395-9408.

45 Y.-H. Chen and C.-S. Yeh, Colloids Surf., A, 2002, 197, 133-139.

46 B. Wiley, Y. Sun, B. Mayers and Y. Xia, Chem. - Eur. J., 2005, 11, 454-463.

47 J. A. Scholl, A. L. Koh and J. A. Dionne, Nature, 2012, 483, 421-427.

48 R. Jin, Nanoscale, 2010, 2, 343-362.

49 J. Xiao and L. Qi, Nanoscale, 2011, 3, 1383-1396.

50 L. Qu, G. Shi, X. Wu and B. Fan, Adv. Mater., 2004, 16, 1200-1203.

51 J. Q. Hu, Q. Chen, Z. X. Xie, G. B. Han, R. H. Wang, B. Ren, Y. Zhang, Z. L. Yang and Z. Q. Tian, Adv. Funct. Mater., 2004, 14, 183-189.

52 S. H. Zhang, Z. X. Xie, Z. Y. Jiang, X. Xu, J. Xiang, R. B. Huang and L. S. Zheng, Chem. Commun., 2004, 9, 1106-1107.

53 D. Yu and V. W. Yam, J. Am. Chem. Soc., 2004, 126, 13200-13201.

54 B. Pietrobon and V. Kitaev, Chem. Mater., 2008, 20, 5186-5190.

55 B.-H. Lee, M.-S. Hsu, Y.-C. Hsu, C.-W. Lo and C.-L. Huang, J. Phys. Chem. C, 2010, 114, 6222-6227.

56 X. He, X. Zhao, Y. Li and X. Sui, J. Mater. Res., 2011, 24, 2200-2209.

57 K.-J. Wu and L. Torrente-Murciano, React. Chem. Eng., 2018, 3, 267-276.

58 S. Zhang, L. Zhang, K. Liu, M. Liu, Y. Yin and C. Gao, Mater. Chem. Front., 2018, 2, 1328-1333.

59 S. Agnihotri, S. Mukherji and S. Mukherji, RSC Adv., 2014, 4, 3974-3983.

60 H. Li, H. Xia, D. Wang and X. Tao, Langmuir, 2013, 29, 5074-5079.

61 J. Yang, H. Yin, J. Jia and Y. Wei, Langmuir, 2011, 27, 5047-5053.

62 J. R. Shimpi, D. S. Sidhaye and B. L. V. Prasad, Langmuir, 2017, 33, 9491-9507.

63 N. Razgoniaeva, M. Yang, P. Garrett, N. Kholmicheva, P. Moroz, H. Eckard, L. Royo Romero, D. Porotnikov, 
D. Khon and M. Zamkov, Chem. Mater., 2018, 30, 1391-1398.

64 L. Yu, H. You, Q. Zhang, L. Zhang and J. Fang, CrystEngComm, 2019, 21, 1529-1533.

65 Y. Wan, Z. Guo, X. Jiang, K. Fang, X. Lu, Y. Zhang and N. Gu, J. Colloid Interface Sci., 2013, 394, 263-268.

66 N. R. Jana, L. Gearheart and C. J. Murphy, Chem. Commun., 2001, 7, 617-618.

67 G. Lee, Mater. Chem. Phys., 2004, 84, 197-204.

68 A. M. Steiner, M. Mayer, D. Schletz, D. Wolf, P. Formanek, R. Hübner, M. Dulle, S. Förster, T. A. F. König and A. Fery, Chem. Mater., 2019, 31, 2822-2827.

69 S. Zhou, J. Li, K. D. Gilroy, J. Tao, C. Zhu, X. Yang, X. Sun and Y. Xia, ACS Nano, 2016, 10, 9861-9870.

70 I. Washio, Y. Xiong, Y. Yin and Y. Xia, Adv. Mater., 2006, 18, 1745-1749.

71 Y. Xiong, I. Washio, J. Chen, H. Cai, Z. Y. Li and Y. Xia, Langmuir, 2006, 22, 8563-8570.

72 T. Zhao, R. Sun, S. Yu, Z. Zhang, L. Zhou, H. Huang and R. Du, Colloids Surf., A, 2010, 366, 197-202.

73 J.-Y. Lin, Y.-L. Hsueh, J.-J. Huang and J.-R. Wu, Thin Solid Films, 2015, 584, 243-247.

74 B. J. Wiley, Z. Wang, J. Wei, Y. Yin, D. H. Cobden and Y. Xia, Nano Lett., 2006, 6, 2273-2278.

75 B. Wiley, Y. Sun and Y. Xia, Acc. Chem. Res., 2007, 40, 1067-1076.

76 A. R. Siekkinen, J. M. McLellan, J. Chen and Y. Xia, Chem. Phys. Lett., 2006, 432, 491-496.

77 N. Grillet, D. Manchon, F. Bertorelle, C. Bonnet, M. Broyer, E. Cottancin, J. Lerme, M. Hillenkamp and M. Pellarin, ACS Nano, 2011, 5, 9450-9462.

78 S. Chen and R. L. Penn, Cryst. Growth Des., 2019, 19, 4332-4339.

79 M. Tsuji, X. Tang, M. Matsunaga, Y. Maeda and M. Watanabe, Cryst. Growth Des., 2010, 10, 5238-5243.

80 Y. Sun, Chem. Soc. Rev., 2013, 42, 2497-2511.

81 K. Eid, H. Wang and L. Wang, Supra-Materials Nanoarchitectonics, 2017, 6, 135-171.

82 I. Pastoriza-Santos and L. M. Liz-Marzán, Nano Lett., 2002, 2, 903-905.

83 Y. Gao, P. Jiang, L. Song, J. X. Wang, L. F. Liu, D. F. Liu, Y. J. Xiang, Z. X. Zhang, X. W. Zhao, X. Y. Dou, S. D. Luo, W. Y. Zhou and S. S. Xie, J. Cryst. Growth, 2006, 289, 376-380.

84 M. Tsuji, M. Ogino, R. Matsuo, H. Kumagae, S. Hikino, T. Kim and S.-H. Yoon, Cryst. Growth Des., 2010, 10, 296-301.

85 A. S. Thakor, J. Jokerst, C. Zavaleta, T. F. Massoud and S. S. Gambhir, Nano Lett., 2011, 11, 4029-4036.

86 N. Li, P. Zhao and D. Astruc, Angew. Chem., Int. Ed., 2014, 53, 1756-1789.

87 L. Y. Chen, C. W. Wang, Z. Yuan and H. T. Chang, Anal. Chem., 2015, 87, 216-229.

88 M. R. Langille, M. L. Personick, J. Zhang and C. A. Mirkin, J. Am. Chem. Soc., 2012, 134, 14542-14554.

89 Y. Zheng, W. Liu, T. Lv, M. Luo, H. Hu, P. Lu, S. I. Choi, C. Zhang, J. Tao, Y. Zhu, Z. Y. Li and Y. Xia, Chem. - Asian J., 2014, 9, 2635-2640.
90 Y. Zheng, X. Zhong, Z. Li and Y. Xia, Part. Part. Syst. Charact., 2014, 31, 266-273.

91 N. R. Jana, L. Gearheart and C. J. Murphy, Adv. Mater., 2001, 13, 1389-1393.

92 B. Nikoobakht and M. A. El-Sayed, Chem. Mater., 2003, 15, 1957-1962.

93 N. R. Jana, L. Gearheart and C. J. Murphy, J. Phys. Chem. B, 2001, 105, 4065-4067.

94 C. J. Murphy, T. K. Sau, A. M. Gole, C. J. Orendorff, J. Gao, L. Gou, S. E. Hunyadi and T. Li, J. Phys. Chem. B, 2005, 109, 13857-13870.

95 J. Zhang, M. R. Langille, M. L. Personick, K. Zhang, S. Li and C. A. Mirkin, J. Am. Chem. Soc., 2010, 132, 14012-14014.

96 T. H. Ha, H.-J. Koo and B. H. Chung, J. Phys. Chem. C, 2007, 111, 1123-1130.

97 L. Chen, F. Ji, Y. Xu, L. He, Y. Mi, F. Bao, B. Sun, X. Zhang and Q. Zhang, Nano Lett., 2014, 14, 7201-7206.

98 H. L. Wu, C. H. Kuo and M. H. Huang, Langmuir, 2010, 26, 12307-12313.

99 P. J. Chung, L. M. Lyu and M. H. Huang, Chem. - Eur. J., 2011, 17, 9746-9752.

100 C.-Y. Chiu, P.-J. Chung, K.-U. Lao, C.-W. Liao and M. H. Huang, J. Phys. Chem. C, 2012, 116, 23757-23763.

101 B.-H. Kuo, C.-F. Hsia, T.-N. Chen and M. H. Huang, J. Phys. Chem. C, 2018, 122, 25118-25126.

102 W. Niu, Y. Duan, Z. Qing, H. Huang and X. Lu, J. Am. Chem. Soc., 2017, 139, 5817-5826.

103 L. Scarabelli, M. Coronado-Puchau, J. J. Giner-Casares, J. Langer and L. M. Liz-Marzan, ACS Nano, 2014, 8, 5833-5842.

104 G. Gonzalez-Rubio, J. Mosquera, V. Kumar, A. PedrazoTardajos, P. Llombart, D. M. Solis, I. Lobato, E. G. Noya, A. Guerrero-Martinez, J. M. Taboada, F. Obelleiro, L. G. MacDowell, S. Bals and L. M. Liz-Marzan, Science, 2020, 368, 1472-1477.

105 M. Hentschel, M. Schaferling, X. Duan, H. Giessen and N. Liu, Sci. Adv., 2017, 3, e1602735.

106 L. Nguyen, M. Dass, M. F. Ober, L. V. Besteiro, Z. M. Wang, B. Nickel, A. O. Govorov, T. Liedl and A. Heuer-Jungemann, ACS Nano, 2020, 14, 7454-7461.

107 D. Seo, J. C. Park and H. Song, J. Am. Chem. Soc., 2006, 128, 14863-14870.

108 C. Li, K. L. Shuford, M. Chen, E. J. Lee and S. O. Cho, ACS Nano, 2008, 2, 1760-1769.

109 Y. Lu, H. Zhang, F. Wu, H. Liu and J. Fang, RSC Adv., 2017, 7, 18601-18608.

110 H. Zhang, Y. Lu, C. Guan, N. Song, Y. Zhang, H. Liu and J. Fang, J. Mater. Chem. C, 2017, 5, 645-653.

111 O. M. Bakr, B. H. Wunsch and F. Stellacci, Chem. Mater., 2006, 18, 3297-3301.

112 C. G. Khoury and T. Vo-Dinh, J. Phys. Chem. C, 2008, 112, 18849-18859.

113 H.-L. Wu, C.-H. Chen and M. H. Huang, Chem. Mater., 2009, 21, 110-114.

114 E. Ye, K. Y. Win, H. R. Tan, M. Lin, C. P. Teng, A. Mlayah and M. Y. Han, J. Am. Chem. Soc., 2011, 133, 8506-8509. 
115 E. Ye, M. D. Regulacio, M. S. Bharathi, H. Pan, M. Lin, M. Bosman, K. Y. Win, H. Ramanarayan, S. Y. Zhang, X. J. Loh, Y. W. Zhang and M. Y. Han, Nanoscale, 2016, 8, 543-552.

116 Z. Li, W. Li, P. H. Camargo and Y. Xia, Angew. Chem., Int. Ed., 2008, 47, 9653-9656.

117 D. Y. Kim, T. Yu, E. C. Cho, Y. Ma, O. O. Park and Y. Xia, Angew. Chem., Int. Ed., 2011, 50, 6328-6331.

118 C. P. Teng, T. Zhou, E. Ye, S. Liu, L. D. Koh, M. Low, X. J. Loh, K. Y. Win, L. Zhang and M. Y. Han, Adv. Healthcare Mater., 2016, 5, 2122-2130.

119 D. Zhu, Y. Liu, M. Liu, X. Liu, P. N. Prasad and M. T. Swihart, J. Mater. Chem. B, 2020, 8, 5491-5499.

120 D. V. Goia and E. Matijević, New J. Chem., 1998, 22, 1203-1215.

121 H. Bönnemann and R. M. Richards, Eur. J. Inorg. Chem., 2001, 2455-2480.

122 C. Burda, X. Chen, R. Narayanan and M. A. El-Sayed, Chem. Rev., 2005, 105, 1025-1102.

123 R. Ferrando, J. Jellinek and R. L. Johnston, Chem. Rev., 2008, 108, 845-910.

124 Y. C. Tsao, S. Rej, C. Y. Chiu and M. H. Huang, J. Am. Chem. Soc., 2014, 136, 396-404.

125 M. S. Akhtar, J. Panwar and Y.-S. Yun, ACS Sustainable Chem. Eng., 2013, 1, 591-602.

126 S. Ahmed, M. Ahmad, B. L. Swami and S. Ikram, J. Adv. Res., 2016, 7, 17-28.

127 P. Khandel, R. K. Yadaw, D. K. Soni, L. Kanwar and S. K. Shahi, J. Nanostruct. Chem., 2018, 8, 217-254.

128 P. Malik, R. Shankar, V. Malik, N. Sharma and T. K. Mukherjee, J. Nanopart., 2014, 2014, 1-14.

129 R. Sankar, P. Manikandan, V. Malarvizhi, T. Fathima, K. S. Shivashangari and V. Ravikumar, Spectrochim. Acta, Part A, 2014, 121, 746-750.

130 H. R. El-Seedi, R. M. El-Shabasy, S. A. M. Khalifa, A. Saeed, A. Shah, R. Shah, F. J. Iftikhar, M. M. Abdel-Daim, A. Omri, N. H. Hajrahand, J. S. M. Sabir, X. Zou, M. F. Halabi, W. Sarhan and W. Guo, RSC Adv., 2019, 9, 24539-24559.

131 D. Alti, M. Veeramohan Rao, D. N. Rao, R. Maurya and S. K. Kalangi, ACS Omega, 2020, 5, 16238-16245.

132 D. P. Yang, X. Liu, C. P. Teng, C. Owh, K. Y. Win, M. Lin, X. J. Loh, Y. L. Wu, Z. Li and E. Ye, Nanoscale, 2017, 9, 15753-15759.

133 M. D. Regulacio, D.-P. Yang and E. Ye, CrystEngComm, 2020, 22, 399-411.

134 D. S. Sheny, J. Mathew and D. Philip, Spectrochim. Acta, Part A, 2011, 79, 254-262.

135 K. Gopinath, S. Kumaraguru, K. Bhakyaraj, S. Mohan, K. S. Venkatesh, M. Esakkirajan, P. Kaleeswarran, N. S. Alharbi, S. Kadaikunnan, M. Govindarajan, G. Benelli and A. Arumugam, Microb. Pathog., 2016, 101, 1-11.

136 T. L. Botha, E. E. Elemike, S. Horn, D. C. Onwudiwe, J. P. Giesy and V. Wepener, Sci. Rep., 2019, 9, 4169.

137 P. Kuppusamy, S. Ilavenil, S. Srigopalram, D. H. Kim, N. Govindan, G. P. Maniam, M. M. Yusoff and
K. C. Choi, J. Inorg. Organomet. Polym. Mater., 2017, 27, 562-568.

138 M. Khan, K. Al-Hamoud, Z. Liaqat, M. R. Shaik, S. F. Adil, M. Kuniyil, H. Z. Alkhathlan, A. Al-Warthan, M. R. H. Siddiqui, M. Mondeshki, W. Tremel, M. Khan and M. N. Tahir, Nanomaterials, 2020, 10, 1885.

139 N. Patra, A. C. Taviti, A. Sahoo, A. Pal, T. K. Beuria, A. Behera and S. Patra, RSC Adv., 2017, 7, 35111-35118.

140 C. Sharma, S. Ansari, M. S. Ansari, S. P. Satsangee and M. M. Srivastava, Mater. Sci. Eng., C, 2020, 116, 111153.

141 P. Hou, H. Liu, J. Li and J. Yang, CrystEngComm, 2015, 17, 1826-1832.

142 D. Kim, J. Resasco, Y. Yu, A. M. Asiri and P. Yang, Nat. Commun., 2014, 5, 4948.

143 S. Liu, G. Chen, P. N. Prasad and M. T. Swihart, Chem. Mater., 2011, 23, 4098-4101.

144 W. Chen, R. Yu, L. Li, A. Wang, Q. Peng and Y. Li, Angew. Chem., Int. Ed., 2010, 49, 2917-2921.

145 S. E. Skrabalak, J. Chen, Y. Sun, X. Lu, L. Au, C. M. Cobley and Y. Xia, Acc. Chem. Res., 2008, 41, 1587-1595.

146 B. Goris, L. Polavarapu, S. Bals, G. Van Tendeloo and L. M. Liz-Marzan, Nano Lett., 2014, 14, 3220-3226.

147 S. W. Chee, S. F. Tan, Z. Baraissov, M. Bosman and U. Mirsaidov, Nat. Commun., 2017, 8, 1-8.

148 Y. Sun and Y. Xia, J. Am. Chem. Soc., 2004, 126, 3892-3901.

149 X. Lu, L. Au, J. McLellan, Z. Y. Li, M. Marquez and Y. Xia, Nano Lett., 2007, 7, 1764-1769.

150 Y. Ma, W. Li, E. C. Cho, Z. Li, T. Yu, J. Zeng, Z. Xie and Y. Xia, ACS Nano, 2010, 4, 6725-6734.

151 E. Gonzalez, J. Arbiol and V. F. Puntes, Science, 2011, 334, 1377-1380.

152 C. H. Tsai, S. Y. Chen, J. M. Song, M. Haruta and H. Kurata, Nanoscale Res. Lett., 2015, 10, 1-9.

153 T. S. Rodrigues, A. G. M. da Silva, A. B. L. de Moura, I. G. Freitas and P. H. C. Camargo, RSC Adv., 2016, 6, 62286-62290.

154 A. G. da Silva, T. S. Rodrigues, T. J. Slater, E. A. Lewis, R. S. Alves, H. V. Fajardo, R. Balzer, A. H. da Silva, I. C. de Freitas, D. C. Oliveira, J. M. Assaf, L. F. Probst, S. J. Haigh and P. H. Camargo, ACS Appl. Mater. Interfaces, 2015, 7, 25624-25632.

155 S. Y. Tee, E. Ye, P. H. Pan, C. J. Lee, H. K. Hui, S. Y. Zhang, L. D. Koh, Z. Dong and M. Y. Han, Nanoscale, 2015, 7, 11190-11198.

156 A. Panacek, L. Kvitek, R. Prucek, M. Kolar, R. Vecerova, N. Pizurova, V. K. Sharma, T. Nevecna and R. Zboril, J. Phys. Chem. B, 2006, 110, 16248-16253.

157 Y. Zhou, Y. Kong, S. Kundu, J. D. Cirillo and H. Liang, J. Nanobiotechnol., 2012, 10, 19.

158 N. M. Zain, A. G. Stapley and G. Shama, Carbohydr. Polym., 2014, 112, 195-202.

159 F. Paladini, M. Pollini, A. Sannino and L. Ambrosio, Biomacromolecules, 2015, 16, 1873-1885.

160 Y. N. Slavin, J. Asnis, U. O. Hafeli and H. Bach, J. Nanobiotechnol., 2017, 15, 65.

161 S. Pal, Y. K. Tak and J. M. Song, Appl. Environ. Microbiol., 2007, 73, 1712-1720. 
162 Z. M. Xiu, Q. B. Zhang, H. L. Puppala, V. L. Colvin and P. J. Alvarez, Nano Lett., 2012, 12, 4271-4275.

163 X. Yan, B. He, L. Liu, G. Qu, J. Shi, L. Hu and G. Jiang, Metallomics: Integrated Biometal. Sci., 2018, 10, 557-564.

164 M. Ramezani, A. Kosak, A. Lobnik and A. Hadela, Text. Res. J., 2019, 89, 5130-5143.

165 S. Tang and J. Zheng, Adv. Healthcare Mater., 2018, 7, e1701503.

166 A. Ivask, A. Elbadawy, C. Kaweeteerawat, D. Boren, H. Fischer, Z. Ji, C. H. Chang, R. Liu, T. Tolaymat, D. Telesca, J. I. Zink, Y. Cohen, P. A. Holden and H. A. Godwin, ACS Nano, 2014, 8, 374-386.

167 G. Franci, A. Falanga, S. Galdiero, L. Palomba, M. Rai, G. Morelli and M. Galdiero, Molecules, 2015, 20, 8856-8874.

168 J. R. Morones, J. L. Elechiguerra, A. Camacho, K. Holt, J. B. Kouri, J. T. Ramirez and M. J. Yacaman, Nanotechnology, 2005, 16, 2346-2353.

169 H. B. Li, P. Liu, Y. Liang, J. Xiao and G. W. Yang, Nanoscale, 2012, 4, 5082-5091.

170 C. M. Goodman, C. D. McCusker, T. Yilmaz and V. M. Rotello, Bioconjugate Chem., 2004, 15, 897-900.

171 P. Nalawade, P. Mukherjee and S. Kapoor, J. Nanostruct. Chem., 2014, 4, 1-8.

172 Y. Wang, J. Wan, R. J. Miron, Y. Zhao and Y. Zhang, Nanoscale, 2016, 8, 11143-11152.

173 M. Banerjee, S. Sharma, A. Chattopadhyay and S. S. Ghosh, Nanoscale, 2011, 3, 5120-5125.

174 N. Cioffi, L. Torsi, N. Ditaranto, G. Tantillo, L. Ghibelli, L. Sabbatini, T. Bleve-Zacheo, M. D'Alessio, P. G. Zambonin and E. Traversa, Chem. Mater., 2005, 17, 5255-5262.

175 L. A. Tamayo, P. A. Zapata, N. D. Vejar, M. I. Azocar, M. A. Gulppi, X. Zhou, G. E. Thompson, F. M. Rabagliati and M. A. Paez, Mater. Sci. Eng., C, 2014, 40, 24-31.

176 M. Raffi, S. Mehrwan, T. M. Bhatti, J. I. Akhter, A. Hameed, W. Yawar and M. M. Ul Hasan, Ann. Microbiol., 2010, 60, 75-80.

177 P. S. Harikumar, Int. J. Sci., 2016, 2, 83-90.

178 R. Lakshminarayanan, E. Ye, D. J. Young, Z. Li and X. J. Loh, Adv. Healthcare Mater., 2018, 7, e1701400.

179 S. Shankar and J.-W. Rhim, Mater. Lett., 2014, 132, 307-311.

180 M. Fleischmann, P. J. Hendra and A. J. McQuillan, Chem. Phys. Lett., 1974, 26, 163-166.

181 G. Demirel, H. Usta, M. Yilmaz, M. Celik, H. A. Alidagi and F. Buyukserin, J. Mater. Chem. C, 2018, 6, 5314-5335.

182 I. Bruzas, W. Lum, Z. Gorunmez and L. Sagle, The Analyst, 2018, 143, 3990-4008.

183 S.-Y. Ding, J. Yi, J.-F. Li, B. Ren, D.-Y. Wu, R. Panneerselvam and Z.-Q. Tian, Nat. Rev. Mater., 2016, 1, 1-16.

184 A. I. Pérez-Jiménez, D. Lyu, Z. Lu, G. Liu and B. Ren, Chem. Sci., 2020, 11, 4563-4577.

185 S. Laing, L. E. Jamieson, K. Faulds and D. Graham, Nat. Rev. Chem., 2017, 1, 1-19.
186 B. Saute, R. Premasiri, L. Ziegler and R. Narayanan, The Analyst, 2012, 137, 5082-5087.

187 K.-Q. Lin, J. Yi, S. Hu, B.-J. Liu, J.-Y. Liu, X. Wang and B. Ren, J. Phys. Chem. C, 2016, 120, 20806-20813.

188 M. J. Seo, G. W. Kim, P. V. Tsalu, S. W. Moon and J. W. Ha, Nanoscale Horiz., 2020, 5, 345-349.

189 C. Wang, B. Liu and X. Dou, Sens. Actuators, B, 2016, 231, 357-364.

190 C. Kuttner, M. Mayer, M. Dulle, A. Moscoso, J. M. LopezRomero, S. Forster, A. Fery, J. Perez-Juste and R. ContrerasCaceres, ACS Appl. Mater. Interfaces, 2018, 10, 11152-11163.

191 R. Kodiyath, S. T. Malak, Z. A. Combs, T. Koenig, M. A. Mahmoud, M. A. El-Sayed and V. V. Tsukruk, J. Mater. Chem. A, 2013, 1, 2777.

192 T. J. Dill, M. J. Rozin, S. Palani and A. R. Tao, ACS Nano, 2016, 10, 7523-7531.

193 J. E. Park, Y. Lee and J. M. Nam, Nano Lett., 2018, 18, 6475-6482.

194 S. He, J. Chua, E. K. M. Tan and J. C. Y. Kah, RSC Adv., 2017, 7, 16264-16272.

195 L. Tian, N. Gandra and S. Singamaneni, ACS Nano, 2013, 7, 4252-4260.

196 C. Liu, L. Tan, L. Li, J. Dong and W. Qian, CrystEngComm, 2017, 19, 3233-3236.

197 J. Li, J. Wu, X. Zhang, Y. Liu, D. Zhou, H. Sun, H. Zhang and B. Yang, J. Phys. Chem. C, 2011, 115, 3630-3637.

198 P. C. Angelomé, H. Heidari Mezerji, B. Goris, I. PastorizaSantos, J. Pérez-Juste, S. Bals and L. M. Liz-Marzán, Chem. Mater., 2012, 24, 1393-1399.

199 Y. Zhang, B. Wang, S. Yang, L. Li and L. Guo, New J. Chem., 2015, 39, 2551-2556.

200 M. Bosman, E. Ye, S. F. Tan, C. A. Nijhuis, J. K. Yang, R. Marty, A. Mlayah, A. Arbouet, C. Girard and M. Y. Han, Sci. Rep., 2013, 3, 1312.

201 H. Guo, F. Ruan, L. Lu, J. Hu, J. Pan, Z. Yang and B. Ren, J. Phys. Chem. C, 2009, 113, 10459-10464.

202 L. Rodríguez-Lorenzo, R. N. A. Álvarez-Puebla, F. J. G. A. de Abajo and L. M. Liz-Marzán, J. Phys. Chem. C, 2010, 114, 7336-7340.

203 S. Lin, X. Lin, Y. Shang, S. Han, W. Hasi and L. Wang, J. Phys. Chem. C, 2019, 123, 24714-24722.

204 Y. C. Cao, R. Jin and C. A. Mirkin, Science, 2002, 297, 1536-1540.

205 D. K. Lim, K. S. Jeon, J. H. Hwang, H. Kim, S. Kwon, Y. D. Suh and J. M. Nam, Nat. Nanotechnol., 2011, 6, 452-460.

206 H. Xu, E. J. Bjerneld, M. Käll and L. Börjesson, Phys. Rev. Lett., 1999, 83, 4357-4360.

207 X. X. Han, B. Zhao and Y. Ozaki, Anal. Bioanal. Chem., 2009, 394, 1719-1727.

208 B. Fazio, C. D’Andrea, A. Foti, E. Messina, A. Irrera, M. G. Donato, V. Villari, N. Micali, O. M. Marago and P. G. Gucciardi, Sci. Rep., 2016, 6, 26952.

209 P. Matteini, M. de Angelis, L. Ulivi, S. Centi and R. Pini, Nanoscale, 2015, 7, 3474-3480. 
210 F. Zhang, N. Wu, J. Zhu, J. Zhao, G.-J. Weng, J.-J. Li and J.-W. Zhao, Sens. Actuators, B, 2018, 271, 174-182.

211 Y. Su, S. Xu, J. Zhang, X. Chen, L. P. Jiang, T. Zheng and J. J. Zhu, Anal. Chem., 2019, 91, 864-872.

212 S. Tian, W. You, Y. Shen, X. Gu, M. Ge, S. Ahmadi, S. Ahmad and H.-B. Kraatz, New J. Chem., 2019, 43, 14772-14780.

213 X. Zhao, M. Deng, G. Rao, Y. Yan, C. Wu, Y. Jiao, A. Deng, C. Yan, J. Huang, S. Wu, W. Chen, T. Lei, P. Xu, W. He and J. Xiong, Small, 2018, 14, e1802477.

214 A. V. Markin, N. E. Markina, J. Popp and D. Cialla-May, TrAC, Trends Anal. Chem., 2018, 108, 247-259.

215 N. E. Markina, S. N. Ustinov, A. M. Zakharevich and A. V. Markin, Anal. Chim. Acta, 2020, 1138, 9-17.

216 N. E. Markina, E. K. Volkova, A. M. Zakharevich, I. Y. Goryacheva and A. V. Markin, Mikrochim. Acta, 2018, 185, 481.

217 Z. Jiang, Q. Zhang, C. Zong, B.-J. Liu, B. Ren, Z. Xie and L. Zheng, J. Mater. Chem., 2012, 22, 18192.

218 C. Zhu, G. Yang, H. Li, D. Du and Y. Lin, Anal. Chem., 2015, 87, 230-249.

219 M. M. Rahman, A. J. Ahammad, J. H. Jin, S. J. Ahn and J. J. Lee, Sensors, 2010, 10, 4855-4886.

220 S. Y. Tee, C. P. Teng and E. Ye, Mater. Sci. Eng., C, 2017, 70, 1018-1030.

221 D. W. Hwang, S. Lee, M. Seo and T. D. Chung, Anal. Chim. Acta, 2018, 1033, 1-34.
222 Y.-G. Zhou, S. Yang, Q.-Y. Qian and X.-H. Xia, Electrochem. Commun., 2009, 11, 216-219.

223 S. Cherevko and C.-H. Chung, Sens. Actuators, B, 2009, 142, 216-223.

224 P. Si, Y. Huang, T. Wang and J. Ma, RSC Adv., 2013, 3, 3487-3502.

225 X. Gao, L. Jin, Q. Wu, Z. Chen and X. Lin, Electroanalysis, 2012, 24, 1771-1777.

226 G. A. El-Nagar, R. M. Sarhan, A. Abouserie, N. Maticiuc, M. Bargheer, I. Lauermann and C. Roth, Sci. Rep., 2017, 7, 12181.

227 P. Miao, B. Wang, J. Yin, X. Chen and Y. Tang, Electrochem. Commun., 2015, 53, 37-40.

228 N. Wang, Y. Han, Y. Xu, C. Gao and X. Cao, Anal. Chem., 2015, 87, 457-463.

229 N. Pariona, A. I. Mtz-Enriquez, D. Sánchez-Rangel, G. Carrión, F. Paraguay-Delgado and G. Rosas-Saito, RSC Adv., 2019, 9, 18835-18843.

230 M. A. Asghar, E. Zahir, S. M. Shahid, M. N. Khan, M. A. Asghar, J. Iqbal and G. Walker, Lwt, 2018, 90, 98-107.

231 S. C. Mali, A. Dhaka, C. K. Githala and R. Trivedi, Biotechnol. Rep., 2020, 27, e00518.

232 S. Jain and M. S. Mehata, Sci. Rep., 2017, 7, 15867.

233 D. H. Nguyen, J. S. Lee, K. D. Park, Y. C. Ching, X. T. Nguyen, V. Phan and T. T. Hoang Thi, Nanomaterials, 2020, 10, 542 . 